\title{
Transcriptome-wide identification of the RNA-binding landscape of the chromatin-associated protein PARP1 reveals functions in RNA biogenesis
}

\author{
Manana Melikishvili ${ }^{1}$, Julia H Chariker ${ }^{2,3}$, Eric C Rouchka ${ }^{3,4}$, Yvonne N Fondufe-Mittendorf ${ }^{1, *}$ \\ ${ }^{I}$ Department of Molecular and Cellular Biochemistry, University of Kentucky, Lexington, KY, USA; ${ }^{2}$ Department of Psycho- \\ logical and Brain Sciences, University of Louisville, Louisville, KY, USA; ${ }^{3}$ Kentucky Biomedical Research Infrastructure Net- \\ work Bioinformatics Core, 522 East Gray Street, Louisville, KY, USA; ${ }^{4}$ Department of Computer Engineering and Computer \\ Science, University of Louisville, Louisville, KY, USA
}

Recent studies implicate Poly (ADP-ribose) polymerase 1 (PARP1) in alternative splicing regulation, and PARP1 may be an RNA-binding protein. However, detailed knowledge of RNA targets and the RNA-binding region for PARP1 are unknown. Here we report the first global study of PARP1-RNA interactions using PAR-CLIP in HeLa cells. We identified a largely overlapping set of 22142 PARP1-RNA-binding peaks mapping to mRNAs, with 20484 sites located in intronic regions. PARP1 preferentially bound RNA containing GC-rich sequences. Using a Bayesian model, we determined positional effects of PARP1 on regulated exon-skipping events: PARP1 binding upstream and downstream of the skipped exons generally promotes exon inclusion, whereas binding within the exon of interest and intronic regions closer to the skipped exon promotes exon skipping. Using truncation mutants, we show that removal of the Zn1Zn2 domain switches PARP1 from a DNA binder to an RNA binder. This study represents a first step into understanding the role of PARP1RNA interaction. Continued identification and characterization of the functional interplay between PARPs and RNA may provide important insights into the role of PARPs in RNA regulation.

Keywords: PAR-CLIP; PARP1; RNA-binding proteins; transcription; alternative splicing

Cell Discovery (2017) 3, 17043; doi:10.1038/celldisc.2017.43; published online 28 November 2017

\section{Introduction}

Poly (ADP-ribose) polymerase 1 (PARP1) or ADPribosyl transferase 1, a multifunctional nuclear protein, belongs to the PARP family of proteins. PARP1 is responsible for initiation, elongation, and branching of ADP-ribose units from donor $\mathrm{NAD}^{+}$molecules onto target proteins, a process known as PARylation. The major target for PARylation is PARP1 itself, but a number of other covalently PARylated proteins have been described, including histones, chromatin remodeling proteins, and transcription factors. PARylation influences the activity of target proteins by modulating protein-nucleic acid interactions, enzymatic activity,

\footnotetext{
*Correspondence: Yvonne N Fondufe-Mittendorf

Tel: +1-859-323-0091; Fax: +1-859-323-5505

E-mail: y.fondufe-mittendorf@uky.edu

Received 9 April 2017; accepted 23 October 2017
}

protein-protein interactions, and/or subcellular localization.

PARP1 was first characterized as a sensor for DNA breaks [1]. Besides its DNA damage response, PARP1 plays a crucial role in regulating numerous molecular processes, such as gene transcription and chromatin remodeling [2-4]. Some of the best functional examples of PARP1 in gene regulation are its regulation of chromatin structure by PARylating histones and destabilizing nucleosomes [5-7], its competition with $\mathrm{H} 1$ for specific target sites [8] and/or its direct interaction with transcription factors and cofactors, such as $\mathrm{NF}-\kappa \mathrm{B}$ or the nuclear factor to activate T-cell gene expression [9-13]. PARP1 also plays critical roles in cell division. For instance, PARP1 regulates components of the mitotic apparatus, such as centromeres and centrosomes, to control microtubule organization during mitosis and chromosome segregation [10]. Taken together, these studies show that PARP1 
exhibits a wide array of subcellular distributions, suggesting a broad and varied role for this protein $[14,15]$.

Although PARP1 has been implicated in multiple regulatory processes, one process for which the paradigm may change is its role in RNA biogenesis. First, PARP1 is known to PARylate poly (A) polymerase (PAP), inhibiting its polyadenylation activity [16], with consequences for pre-mRNA splicing regulation. Second, PARP1 binds to noncoding pRNAs to silence rDNA chromatin [17]. Third, PARP1 PARylates heterogeneous nuclear ribonucleoproteins (hnRNPs), which play important roles in pre-mRNA splicing and translation regulation [18]. Fourth, we recently identified PARP1 as an mRNA-binding protein $[19,20]$, providing further evidence that PARP1-/PARylationmediated events function directly to control premRNA processing. These findings serve to define PARP1 as a co-transcriptional splicing regulator [20]. One possible mechanism for this co-transcriptional function is that PARP1 acts as an adapter, bringing RNA close to chromatin [20]. In fact, a widespread association of chromatin-binding proteins with RNA was shown in vivo, supporting the idea of cotranscriptional RNA splicing [21].

We previously identified PARP1 as a novel RNAbinding protein (RBP) using photoactivatableribonucleoside-enhanced crosslinking and immunoprecipitation (PAR-CLIP). This study raised the interesting possibility that PARP1 plays crucial roles in many aspects of RNA processing to alter gene expression via regulation of $\mathrm{mRNAs}$. Taken together, the identification and characterization of PARP1 mRNA interactions may provide important insights into the role of PARP1 in mRNA regulation and subsequent human disease. However, the breadth, range, and functional location of mRNA types bound by PARP1 has not been explored. In order to identify the direct RNA targets and precise binding sites of PARP1 protein in vivo, we again applied PAR-CLIP followed by deep sequencing (PAR-CLIP-seq). This method is known for its precise identification of binding sites resulting from $\mathrm{T}$-to-C sequence conversions upon RNA-protein crosslinking. We observed that PARP1 was predominantly crosslinked to mRNAs. PAR-CLIP-binding regions contained guanine-cytosine-rich sequences, and RNA-protein interaction was further confirmed by gel mobility-shift assays. Furthermore, we narrowed down the RNA-binding region of the PARP1 protein. The enrichment of many other mRNA-binding proteins (mRBPs) among the large number of PARP1-mRNA targets suggests that PARP1 has a broad role in the regulation of many genes. A continuous identification and characterization of functional interplay between PARPs and RNA may provide important insights into the role of PARPs in RNA regulation.

\section{Results}

\section{PARP1 binds distinct coding and ncRNA sequences}

In our previous experiments we established for the first time that PARP1 binds to RNA in vivo [20]. In the present study, we expanded on previous studies [20, 22] to identify PARP1-RNA targets utilizing the PARCLIP-seq method [23-26] (Figure 1a) in human HeLa cells. Following UV crosslinking, PARP1-bound RNAs were immunoprecipitated under stringent conditions. Radiolabeled PARP1-bound RNA complexes were separated by NuPAGE and observed using a Phosphorimager (Figure 1b). To ensure that only PARP1 protein-bound RNAs were used for further analysis, gels were transferred onto nitrocellulose membranes, visualized by autoradiography (Figure 1c), and the presence of PARP1-bound RNAs was confirmed by western blot analysis (Figure 1d). The results from these experiments demonstrate the robustness and specificity of the PARP1-RNA complexes identified by PAR-CLIP (Figure 1).

In the analysis of the phosphorimages of the radiolabeled PARP1-RNA complexes, we observed two major bands, one migrating at $\sim 100 \mathrm{kDa}$ and the other migrating at $\sim 140 \mathrm{kDa}$. This $\sim 140-\mathrm{kDa}$ band based on the estimation from the protein standard we used is indeed PARP1 (Using other protein standards, this band runs according to PARP1's predicted molecular weight of $\sim 116 \mathrm{KDa}$ - see Supplementary Materials and Methods). Indeed, this band was later confirmed by western blot analysis as PARP1-bound RNA (Figure 1d). Stringent digest with RNase T1 resolved the PARP1-RNA bands to within the estimated molecular weight of PARP1 $\sim 140 \mathrm{kDa}$ (Supplementary Figure S1a). The 100-kDa band contains cleaved PARP1 as identified with antibody that recognizes the c-terminal domain of PARP1 (data not shown). In addition to these two bands, we also observed signals from a higher molecular weight complex ( $>260 \mathrm{kDa}$ ), possibly due to larger complexes that did not migrate into the gel (Supplementary Figure 1a). We suspect that this band likely represents other abundant RNA binding near PARP1binding sites, or PARP1 crosslinked to longer target RNA segments [27] (Figure 1b-d). This interpretation is reasonable, given that similar trends have been observed with other RNA-binding proteins [28]. 
a

PARP1 PAR-CLiP

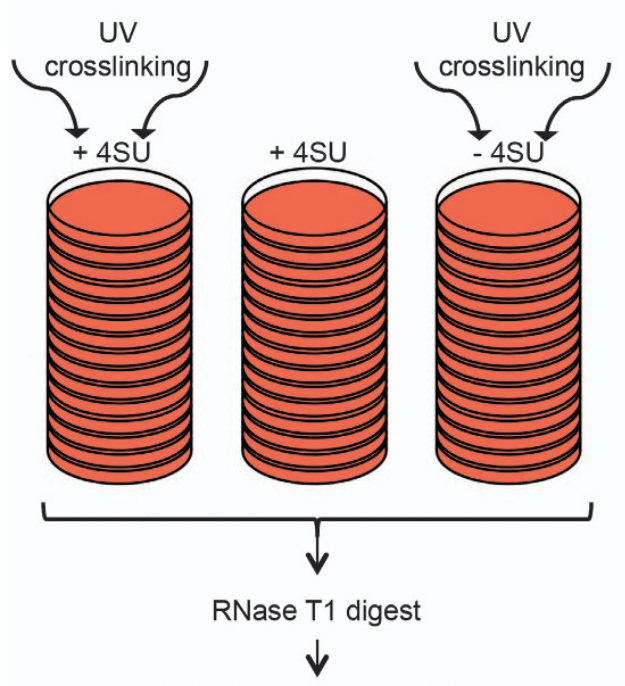

PARP1 immunoprecipitation

$\downarrow$

Isolate and sequence RNA b

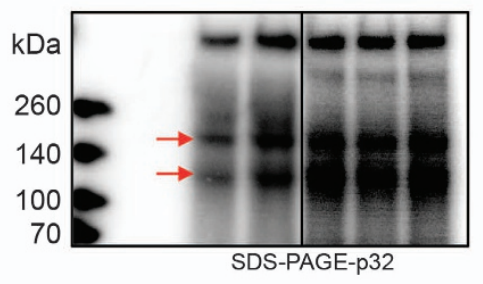

c

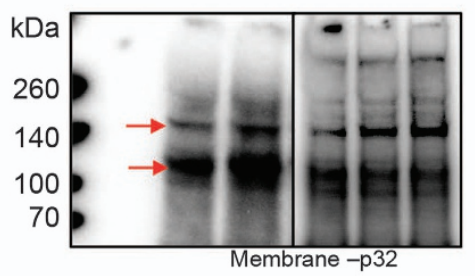

d

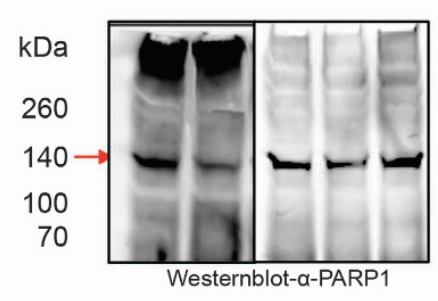

f

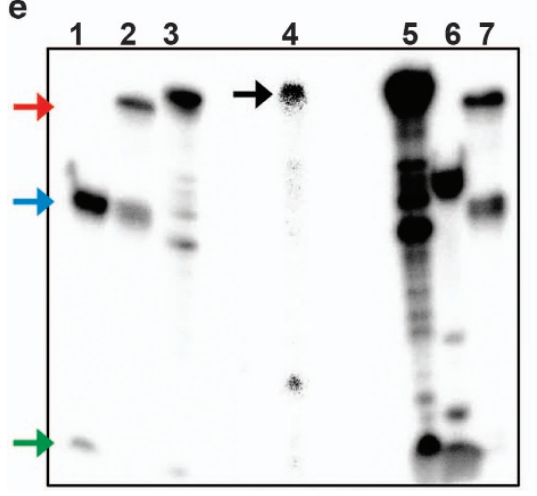

Gel purification

RT \& PCR

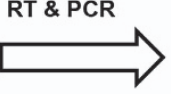

Figure 1 Processing of PAR-CLIP RNA samples for sequencing. (a) Outline of experiments. PAR-CLIP of endogenous PARP1 was performed on HeLa cells using 4-thiouridine (4SU). (b) PAR-CLIP samples run on an SDS PAGE gel were imaged with Typhoon. (c) Protein-bound samples were transferred onto a nitrocellulose membrane and exposed to a phosphoimager screen and imaged using the Typhoon. (d) The same membrane in a was probed for the presence of PARP1-bound RNAs using an antibody to PARP1. Red arrows (b-d) indicate PARP1 bound-RNA—one at $\sim 140 \mathrm{kDa}$ and a shorter fragment at $\sim 100 \mathrm{kDa}$. As the antibody recognizes the larger fragment, we considered this as the full-length protein. The lower band could be a proteolytic fragment as determined by mass spectrometry, lacking the $\mathrm{N}$ terminus, and rendering it undetectable by the antibody raised against the N-terminal domain of the protein. (e) Processing of PARP1-bound RNAs for sequencing. A representative denaturing (8 M urea) polyacrylamide gel showing the different steps of adapter ligation to RNA samples. PARP1-bound RNAs were eluted from membrane in c, deproteinized, ligated to $3^{\prime}$ and $5^{\prime}$ adapters (lane 4). Lanes 1 and 6 are control 19-mer and 24-mer labeled RNAs, ligated to $3^{\prime}$ adapter. These ligated 3' adapter control RNAs were further ligated to $5^{\prime}$ adapters (lanes 2 and 7 ). The green arrow indicates unligated 19-mer and 24-mer RNAs (Lanes 1 and 6, respectively); the blue arrow indicates 3'-adapter ligated control RNAs; the red arrow indicates $3^{\prime}$ adapter and 5'-adapter ligated control RNAs. These controls were used to test the ligation efficiency of our samples. The black arrow (lane 4) indicates $3^{\prime}$ and 5' adapter ligated PARP1-bound RNA samples. (f) Adapter-ligated samples were subjected to limited PCR amplification. Lanes 1 and 2 show $3^{\prime}$ and 5' ligated 19-mer and 24-mer control RNAs converted to cDNA and PCR-amplified. Lanes 3 and 4 are the PARP1-bound RNAs subjected to cDNA conversion and PCR amplification. The black arrow shows the PCR products used for sequencing. 
To validate the specificity of PARP1-RNA binding, we performed several control experiments. (1) A control PAR-CLIP experiment using nonspecific antibodies ( $\mathrm{IgG})$ to precipitate RNA complexes failed to detect any RNA (not shown). (2) Cells not treated with thiouridine or non-crosslinked cells failed to

a

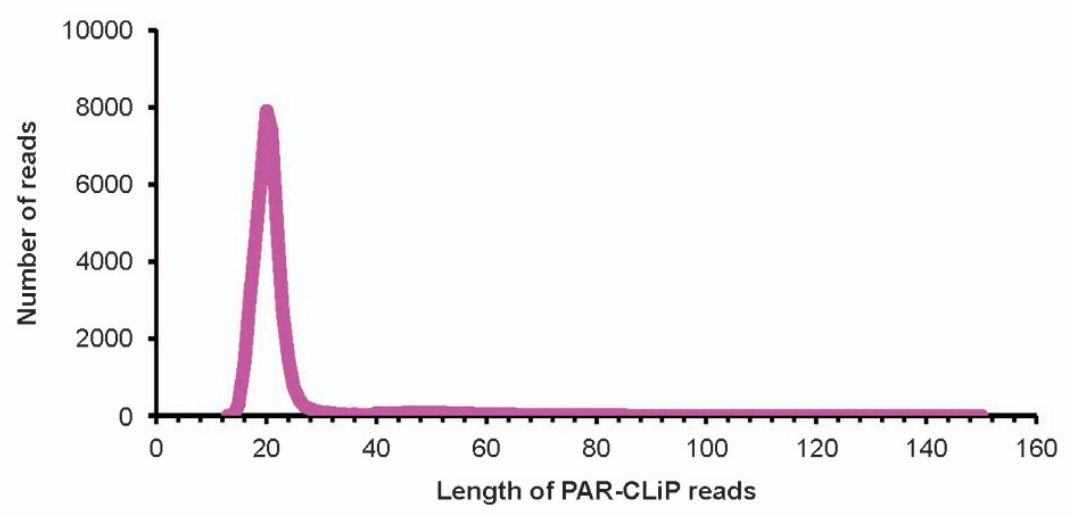

b

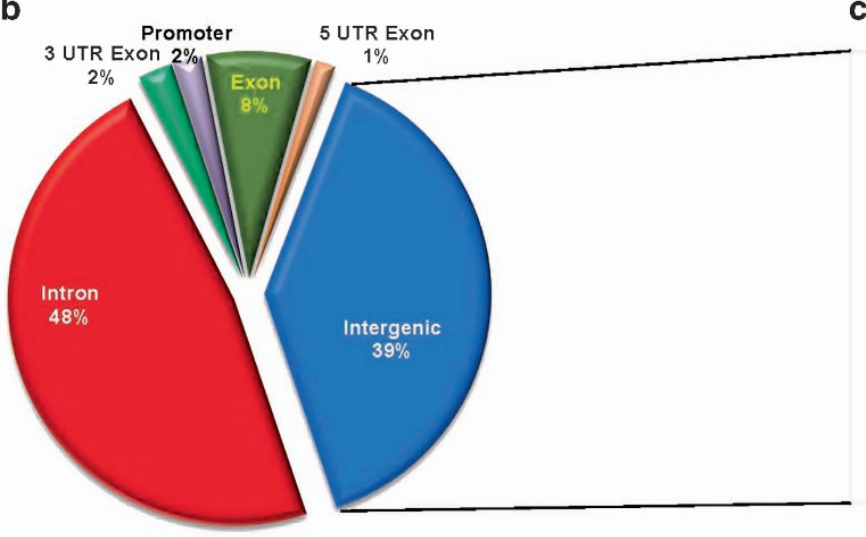

C

d

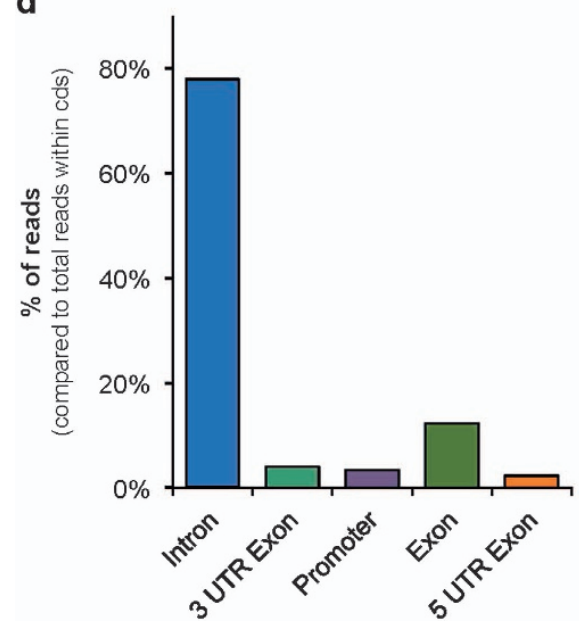

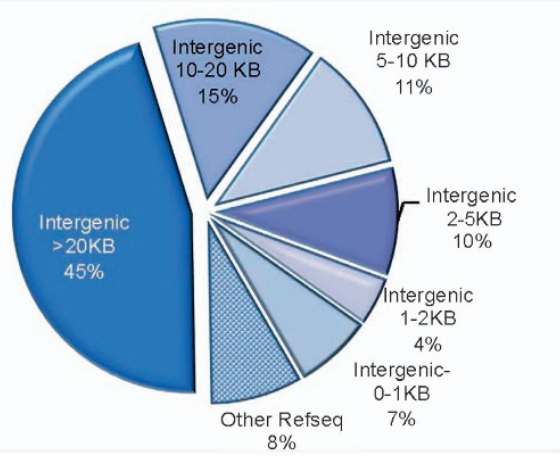

e

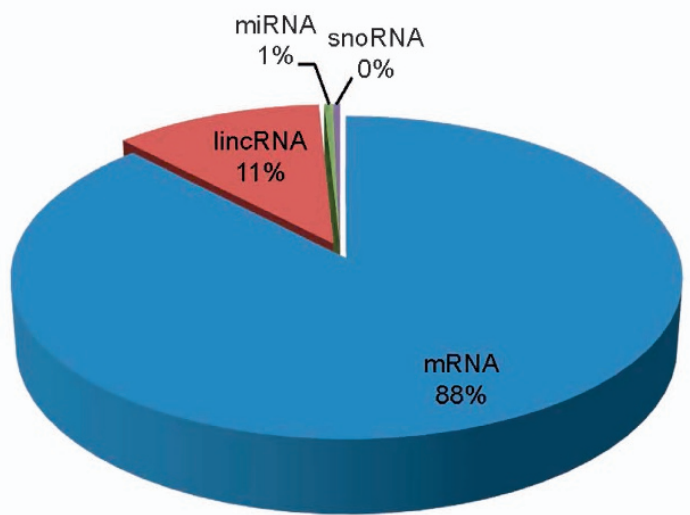

Figure 2 PARP1 RNA binding characteristics. Analyses of PARP1 PAR-CLIP-tags. (a) Fragment length distribution of the PARPCLIP-reads. Insert Table 1 shows the length and number of PARP1 PAR-CLIP sequences. (b) The pie chart shows PARP1-PARCLIP peaks. Most PARP1-CLIP tags mapped to intronic regions, indicating PARP1 binding to mRNA. The intron enrichment is consistent with previous reports that PARP1 binds mainly nascent transcripts. (c) Further analyses of the intergenic regions show other possible regions that are regulatory. (d) Distribution of PARP1 PAR-CLIP tags within gene regions. (e) Distribution of PARP1-PAR-CLIP reads within different types of RNA. 
Table 1 Length of PARP1-PAR-CLIP reads

\begin{tabular}{lc}
\hline k-mer & Number of reads \\
\hline 16 -mer & 2142 \\
$17-$ mer & 3897 \\
18 -mer & 5271 \\
$19-$ mer & 6894 \\
$20-$ mer & 8935 \\
$21-$ mer & 5883 \\
$22-$ mer & 3535 \\
$23-$ mer & 1888 \\
\hline
\end{tabular}

Abbreviations: PAR-CLIP, photoactivatable-ribonucleoside-enhanced crosslinking and immunoprecipitation; PARP1, Poly (ADP-ribose) polymerase 1.

immunoprecipitate a significant amount of PARP1bound RNA (Supplementary Figure S1b, lanes 2 and 3 , respectively), although PARP1 protein remained efficiently precipitated as determined by western blot analysis of the immunoprecipitated complexes (Supplementary Figure S1c, lane 5, bottom; Supplementary Figure 1d, Lanes 3 and 5, bottom). (3) Experiments with stringent RNaseA treatments eliminated the PARP1-RNA bands (Supplementary Figure S1b, Lanes 6 and 7, Supplementary Figure S1e). (4) Knockdown of PARP1 abolished the PARP1-RNA band (Supplementary Figure S1c, lane 6). (5) Lastly, treatment of cells with PJ34 (PARylation inhibitor) for 1 or $24 \mathrm{~h}$ did not change the PARP1RNA-binding profile (Supplementary Figure S1c, lanes 3 and 4), suggesting that this binding is specific for PARP1 and not PAR.

After confirming PARP1-RNA binding, the PARP1-RNA complexes were cut from the membrane, eluted, deproteinized, purified, and ligated to adapters (Figure 1e). The resulting ligated RNAs were converted to cDNA followed by limited PCR amplification experiments (Figure if and Supplementary Figure S2a). Initially, these PCR fragments were cloned into TOPO-blunt vector, checked for correct insert size by restriction enzyme digest (Supplementary Figure S2b), and Sanger-sequenced. From these pilot experiments, the mean fragment length was 21 nucleotides (from the main $140 \mathrm{kDa}$ PARP1-RNA band), 31 nucleotides (from the $200 \mathrm{kDa}-\mathrm{PARP1}-$ RNA fragment), and 7 nucleotides from ( $70 \mathrm{kDa}-$ PARP1-RNA band; Supplementary Figure S2c). For subsequent studies, only the bands resulting from the main PARP1-bound RNA bands $(\sim 140 \mathrm{kDa})$ were used. Seven biological replica experiments were performed, barcoded, and pooled for sequencing using PE Illumina sequencing on a HiSeq 2500. From the various biological replicate experiments, we obtained $0.9-97 \times 10^{6}$ reads after sequencing (Supplementary Table S1). These sequences were subsequently trimmed from adapter sequences yielding a total of 0.6 $39 \times 10^{6}$ unique reads, $47 \%$ of which mapped to the human genome (hg38) allowing 0-2 mismatches.

Next, we grouped them by overlaps using the PARalyzer software [29]. The identified segments of RNA represented peaks of T-to-C conversion (binding sites), with a mean length of 21 nucleotides (mean and mode of $20 \mathrm{nt}$ ) from uniquely aligned T-to-C reconciled reads (Figure 2a and Table 1). Groups of overlapping PAR-CLIP sequence reads were considered binding sites if they (1) passed thresholds of $\geq 0.25$ for T-to-C conversion frequency, (2) contained more than five reads with $\mathrm{T}$-to-C conversion (one mismatch maximum allowed per read), and (3) showed at least two independent $\mathrm{T}$-to- $\mathrm{C}$ conversions. Biological replicates, although with different sequencing depth, showed similar binding patterns (Supplementary Figure S3).

To identify PARP1-RNA target sites, we analyzed the distribution of PAR-CLIP tags in the human genome by defining six regions (exon, introns, promoter, $5^{\prime}$ UTR, 3' UTR, and intergenic regions). The distribution of binding sites across individual transcripts provided insights into PARP1 targeting. Approximately $48 \%$ of PAR-CLIP peak tags (see Materials and Methods) mapped to introns, $\sim 8 \%$ mapped to exons, $2 \%$ to promoter regions, $2 \%$ to $3^{\prime}$ UTR, $1 \%$ to $5^{\prime}$ UTR, and $39 \%$ mapped to intergenic regions (Figure $2 \mathrm{~b}$ ). The over-representation of intronic PAR-CLIP reads indicates that PARP1 binds pre-mRNAs (nascent transcripts) and is consistent with our hypothesis that PARP1 plays a role in pre-mRNA splicing and processing. On the other hand, the observation of a high percentage of PARP1-PAR-CLIP reads to intergenic regions suggests the possibility that these PAR-CLIP tags may correspond to previously unidentified isoforms of genes with alternative terminal exons. To test this idea, we carried out two types of analyses. First, we examined the distance between intergenic clusters and neighboring RefSeq genes. An exponential increase in the cumulative number of tags within $10 \mathrm{~kb}$ downstream of known stop codons compared to linear increases beyond $10 \mathrm{~kb}$ was detected. For instance, $39 \%$ of these intergenic peaks mapped within $10 \mathrm{~kb}$ of the nearest stop or start codon, respectively (Figure 2c and Supplementary Table S2). This suggests that in addition to binding known $3^{\prime}$ UTRs (Figure 2b), PARP1 binds to unannotated $3^{\prime}$ UTR extensions of known genes (Supplementary Figure S4). Second, we asked whether the remaining intergenic reads map to 
genes annotated in other reference genomes, as determined from the 'RefSeq Other' track in the UCSC genome browser. We observed that $8 \%$ mapped to genes annotated within other RefSeq genomes. These analyses show that by doing a more detailed analyses only $\sim 18 \%$ ( $45 \%$ of the initial $39 \%$ intergenic reads as shown in Figure 2b) of PARP1-PAR-CLIP tags map to intergenic regions (Figure $2 \mathrm{c}$ and Supplementary Figure S4).

\section{$m R N A$ is the major species bound by PARPI}

Next, we analyzed the distribution of PARP1-PARCLIP reads in coding regions. This analysis showed that $\sim 78 \%$ of the reads mapped to introns (Figure $2 \mathrm{~d}$ ), raising the possibility that PARP1 contributes to the recognition of specific intronically encoded RNAs such as mRNAs, microRNAs, small nuclear RNAs, and heterogenous RNAs, and influences the rates of various competing RNA processing steps. To examine this, we analyzed the types of RNAs bound by PARP1 from the PAR-CLIP data. Our analyses show that most of the PAR-CLIP peaks were within mRNAs (88\%) compared to the other RNA types, demonstrating that mRNA is the major substrate of the PARP1-RNA complex. On the other hand, crosslink sites were also detected in different classes of RNAs: 2870 peaks (or $11 \%$ of total RNAs bound) in long intergenic noncoding RNAs, 124 peaks (or $1 \%$ of the total RNAs bound) in microRNAs, and 88 peaks within small nuclear RNAs (Figure $2 \mathrm{e}$ and Supplementary Table S3). These results suggest possible novel functions for PARP1 in the regulation of the metabolism of other RNAs as well.

As an alternative method to validate these binding sites, we performed formaldehyde-crosslink RNA immunoprecipitation with nuclear extracts [30]. Enrichment of candidate RNAs was similarly observed using this method (Supplementary Figure S5). Combined, these data support the specificity of PARP1PAR-CLIP-seq and suggest that our observed interactions are indeed interactions between PARP1 and RNA.

\section{RNA motifs bound by PARP1}

We next asked whether PARP1 binds to a particular RNA sequence motif. For that, we applied cERMIT [31] to define the in vivo RNA recognition element for PARP1. The three highest-scoring motifs were generally GC-rich (Figure 3a); this nucleotide composition was observed regardless of the mRNA region of the identified PAR-CLIP tags (Figure 3a). Failure to determine a highly conserved binding motif prompted us to use an unbiased k-mer approach to determine the enrichment of specific sequences within PAR-CLIP data. For this, the 2-nt PARP1-PAR-CLIP data set surrounding the crosslink sites was compared to the genome as a whole to identify k-mers enriched in PARP1-PAR-CLIP reads. Our choice of k-mers allowed us to detect smaller localized signals than cERMIT, which begins with 5-mer seed regions. Starting with 3-mers, we observed an enrichment of GC-rich 3-mers (data not shown). However, as RNA recognition elements are typically longer than 3-mers, we performed further analyses using 4-mers. Again, this analysis showed an enrichment of GC-rich 4-mers (Figure 3b), whereas AT-rich 4-mers were depleted (Figure 3c and d). We repeated the analyses with 6mers and 8-mers, and clearly the enriched k-mers were GC-rich k-mers, although these longer GC-rich are interspersed by AT k-mers (Supplementary Figure S6). Our data show that PARP1 protein RNA-binding sites were comparatively GC-rich, suggesting a tolerance for these GC-rich residues, whereas AT-rich residues were relatively less well tolerated. This information is of interest as during PAR-CLIP experiments G-containing sequences are normally trimmed by RNase T1, and the only way for these guanosines to survive this cleavage is if they are protected by direct binding of the PARP1 or by stable RNA secondary structure [32]. Our results therefore suggest that PARP1 binds to GCrich regions and protects these G-rich regions from RNase T1 cleavage.

\section{Splicing and gene expression changes in response to PARP1 loss}

To test whether transcripts bound by PARP1 are affected upon PARP1 depletion, we determined the global patterns of PARP1-dependent transcription/ splicing changes. For this, cells were transfected with ONTARGETplus short interfering RNA (siRNA) targeting PARP1 and for control experiments with non-targeting siRNAs. Depletion of PARP1 protein was confirmed by western blot analyses, which showed an $\sim 70 \%$ reduction in PARP1 protein levels in the knockdown cells (Figure 4a). Total RNA was isolated from control non-targeting siRNA and PARP1 knockdown (KD) cells, and poly(A)-selected mRNA sequencing was performed on the Illumina platform. Biological replicas from RNA-seq showed high Pearson correlation (Supplementary Table S4), allowing pooling of samples for further analyses. First, we measured changes in gene expression at the transcript level due to PARP1 knockdown. We identified 217 significantly upregulated and 81 downregulated genes, 
a

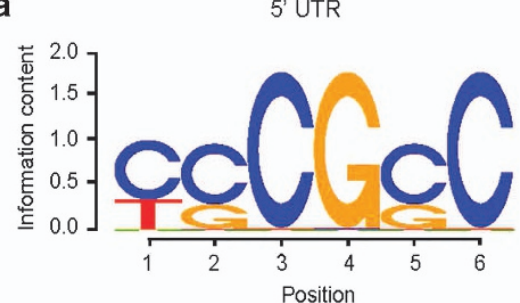

b
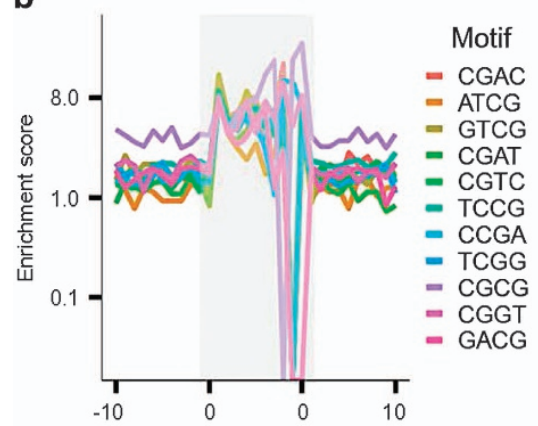
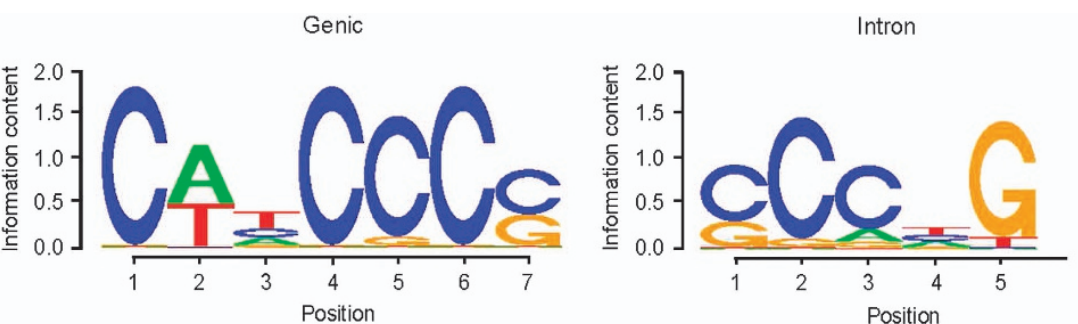

C

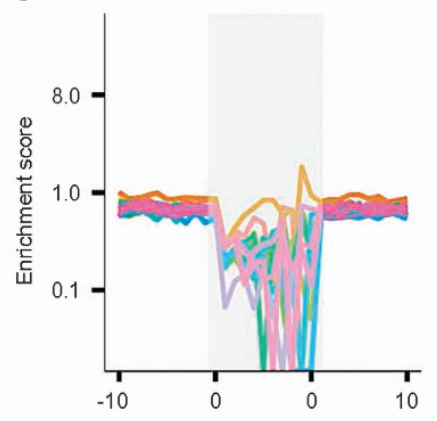

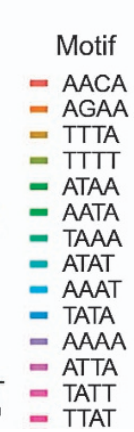

d

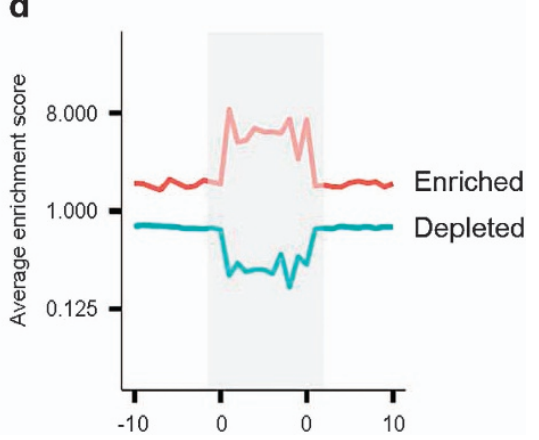

Figure 3 Sequence-binding characteristics of PARP1-bound RNAs. (a) Logo plots of some of the motifs found in the various PARP1-binding regions as determined by CERMIT. (b) Enriched 4-mer motifs found within percentile regions of the PARP1binding site (gray) along with their enrichment scores in the flanking upstream and downstream regions. (c) Depleted 4-mer motifs within the PARP1-binding site (gray) along with their enrichment scores in the flanking upstream and downstream regions. The enrichment scores for $\mathbf{b}, \mathbf{c}$ are determined by the log2 odds score of the frequency of the motif in the region versus the frequency of the motif in the genome. (d) Average enrichment score for enriched and depleted motifs from (b, c), respectively.

including PARP1 (using a cutoff of twofold expression and $P$-value of 0.05 versus non-targeting control; Supplementary Table S5). GO analysis using Gene Set enrichment analysis (GSEA) showed that the top biological processes targeted by the genes upregulated in PARP1 KD cells are NMD, translation, protein metabolism, selanocysteine synthesis, and gene expression. Genes that were downregulated in PARP1 knockdown cells are involved in RNA-binding and poly-A-RNA-binding using GSEA (Figure 4b). We next compared PARP1 RNA targets to genes affected by PARP1 knockdown, and did not observe any meaningful correlation between genes that were bound and trends in gene expression changes. Nevertheless, we observed that $\sim 29 \%$ of genes transcripts affected by PARP1 knockdown were also bound by PARP1 in our PAR-CLIP analysis (Figure 4c).

Our previous study in Drosophila cells suggested that PARP1 plays a role in alternative splicing regulation [20]. In order to assess the effect of PARP1 in splicing, we also analyzed the RNA-seq data for differential alternative splicing events. Using stringent criteria to identify changes in alternative splicing events, we showed that PARP1 depletion resulted in changes in alternative splicing for 791 genes. These changed events included mutually exclusive exons (42.4\%), skipped exon $(25.6 \%)$, retained intron $\left(4.2^{\%}\right)$, alternative $5^{\prime}$ splice site $(23.5 \%)$, and alternative $3^{\prime}$ splice site $(4.4 \%$; Figure $4 d)$. We validated some of these changes in alternative splicing due to PARP1 depletion using qRT-PCR (Supplementary Figure S7). The number of alternatively spliced genes are slightly lower than those observed in our previous studies with Drosophila, where we observed many more changes in alternative splicing [20]. We attribute this low number to possible redundancy with other PARP proteins in humans. GO molecular function terms as determined using GSEA for the targeted alternative spliced genes include nucleosome binding, Poly-A-binding, and RNA binding (Figure 4e).

\section{Positional effects of PARP1 in splicing regulation}

To extend the analysis of the role of PARP1 in alternative splicing, we averaged the presence of PARP1 PAR-CLIP reads along all exon/intron and intron/exon boundaries, representing $3^{\prime}$ and $5^{\prime}$ splice sites, respectively. PARP1 binds uniformly within introns, whereas its binding is enriched at the ends of exons - specifically within 50 nucleotides upstream of start of the exon and 50 nucleotides downstream of the end of the exon (Figure 5a). The observed exon bias reflects the distribution of binding sequences 
a

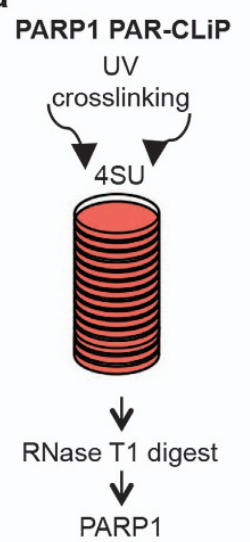

immunoprecipitation

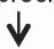

Isolate and sequence RNA

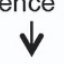

PAR-CLiP

computational pipeline

$\downarrow$

Binding sites

and target genes

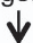

Compare mRNA changes for PARP1 mRNA targets and non-targets

d

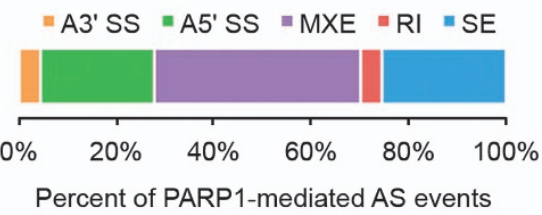

b
$25 \mathrm{nM}$

Con siRNA

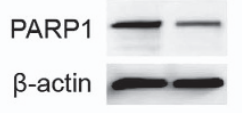

- TTTTT

AAAAA AAAAA $\downarrow$

Computational pipeline

mRNA changes

1. Transcription

2. Splicing
PARP1 knock down

\section{hanges
cription
ch}

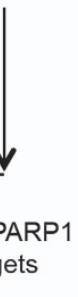

e

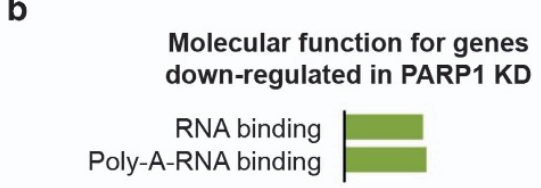

Molecular function for genes up-regulated in PARP1 KD

Gene expression Metabolism of proteins Nonsense mediated decay Selenocystein synthesis SRP-dependent Eukaryotic translation

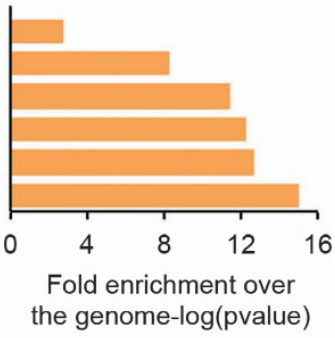

C PARP1 PAR-CLiP/RNA-seq data intersection
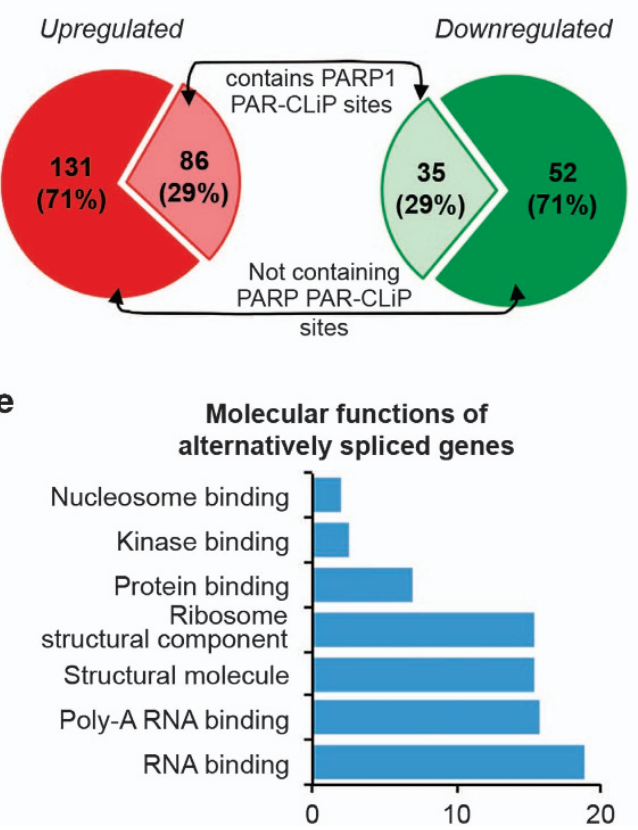

Figure 4 Loss of PARP1 targeted transcriptional and splicing regulation. (a) Experimental and computational workflow for analyzing the intersection of RNAs bound by PARP1 and RNAs whose expression is mediated by PARP1. Left side shows PARCLIP workflow, whereas the right side shows RNA-seq workflow after PARP1 depletion (b). Gene Ontology (Molecular function terms) for genes with significantly changed expression in PARP1 knockdown cells. (c) Proportion of genes overlapping in combined RNA-seq/PARP1-PAR-CLIP data sets. (d) Percentage of the alternative splicing events identified as PARP1 mediated with PARP1 knockdown: alternative 3' splice site (orange); alternative 5' splice site (green); mutually exclusive exons (purple); retained intron (pink); skipped exon (blue). (e) Gene Ontology analysis (molecular function terms) of the genes identified as PARP1-regulated at the splicing level.

within target RNAs and suggests that PARP1 binds mRNA. Although we had observed PARP1 PARCLIP reads in introns (Figure $2 b$ ), the density of these reads at exon-intron boundaries suggests a functional role of PARP1 in demarcating exons. Thus, the binding of PARP1 preferentially at exonic sequences, especially upstream of $5^{\prime}$ and $3^{\prime}$ of splice sites, is consistent with the model that proteins that regulate splicing bind pre-mRNA at functional regions.

To better understand the impact of PARP1 in splicing, we combined PAR-CLIP data with the analysis of 
splicing profiles upon PARP1 depletion to determine the position-dependent regulatory effects of PARP1RNA interactions. To this end, we analyzed the rMATS outputs for skipped exon events using the bioinformatics software rMAPS [33], which systematically generates RNA maps for the identification of position-dependent effects of RNA-binding proteins. The rMAPS program is extremely useful for the computational detection of binding sites around differential alternative splicing events for over 100 of known RBPs. Using the rMAPS-based analysis (with default parameters), along with the list of all PARP1 PAR-CLIP peaks and detected skipped exon events, we identified binding patterns of PARP1 within the PARP1-dependent alternatively spliced exons (Figure $5 b$ ). Restricting the analyses to only significant exon-skipping splice events, we found that for those enhanced and included exons, there is a significant PARP1 binding occurring about $125 \mathrm{bp}$ downstream of the adjacent $5^{\prime}$ exon, and binding occurring about 250 bp upstream of the adjacent $3^{\prime}$ exon (peaks in red). If the exon is excluded, there is a significant binding of PARP1 within the exon itself (in blue) as well as within the upstream and downstream introns. Although it is possible that factors related to translational efficiency and/or RNA stability may affect the regulatory landscape of PARP1-responsive splicing events, the differential expression of the PARP1 together with the enrichment of PARP1-binding and its positional enrichment relative to the regulated exons suggests that many or most of the identified skipped exon splicing events are likely direct targets.

\section{Biochemical characterization of $P A R P 1$ protein-RNA- binding sites}

PARP1 encompasses several functional domains: three zinc-finger domains (Zn1, 2, and 3), a nuclear localization signal region, a breast cancer suppressor protein-1 domain (BRCT), a WGR domain (automodification domain), and the catalytic PARP domain (Figure 6a). To begin to understand PARP1-RNA binding, we purified recombinant full-length human PARP1 (PARP1-FL) and truncated mutants lacking the C-terminal catalytic active site $(\triangle \mathrm{CAT})$, the DBD - the first two zinc fingers $(\Delta \mathrm{Zn} 1 \mathrm{Zn} 2)$; the third zincfinger domain $(\Delta \mathrm{Zn} 3)$, the automodification domain $(\Delta \mathrm{WGR})$, or the protein-protein interaction domain ( $\triangle \mathrm{BRCT}$ domain) from bacterial cells (Figure $6 \mathrm{~b}$ ). Their presence was confirmed through western blot analyses using PARP1 antibody (lanes 1-6, respectively; Figure 6c) and their proper folding confirmed using circular dichroism spectroscopy analyses
(Supplementary Figure S8a). We addressed whether PARP1-RNA direct binding is dependent on other factors, such as contaminating DNA and/or PARP1 PARylation. First, recombinant PARP1-FL was incubated with a radiolabeled synthetic 19-mer ssRNA (chrom15: 53554024-53554044) corresponding to one of the binding sites identified by PAR-CLIP. The protein-RNA complexes were then resolved on a native polyacrylamide gel (Figure 6d and Supplementary Figure S8b). A supershift corresponding to PARP1-RNA complex was observed (Supplementary Figure S8b, lane 2). Second, the PARP1-RNA complex was treated either DNase1 or RNaseA, confirming that RNA is the nucleotide species bound by PARP1 as DNase1 treatment did not change the binding profile but RNaseA completely digested the RNA (Supplementary Figure S8b, lanes 5 and 6, respectively). In addition, treatment of PARP1 with PJ34 did not inhibit PARP1 binding to RNA (Supplementary Figure S8b, lane 4), whereas PARylation of PARP1 by NAD+ abolished its RNAbinding (Supplementary Figure 8b, Lane 3), indicating that PARP1-RNA binding is due to PARP1 and not PAR. As a control, RNA was incubated with increasing amounts of bovine serum albumin and no significant shift in RNA mobility was observed (data not shown).

We next asked which domain of PARP1 is required for its PARP1-RNA binding. EMSA was performed using PARP1-FL as well as truncated mutants by individually incubating them with the radiolabeled synthetic 19 mer RNA (as above; Figure 6d). As seen previously, discrete shifted bands corresponding to PARP1-RNA complexes were observed for all the proteins tested. We then determined the binding affinities of PARP1-FL and mutants to RNA by performing EMSA, incubating $0.05 \mu \mathrm{M}$ radiolabeled 19-nt RNA with increasing concentrations $(0-2.5 \mu \mathrm{M})$ of PARP1-FL or truncated proteins (Figure $7 \mathrm{a}-\mathrm{f}$ ). The fraction bound to total RNA as a function of increased protein concentration for each protein was used to calculate the affinity of that particular protein for RNA (Figure $7 \mathrm{~g}$ and Supplementary Figure S9). Interestingly, these proteins bind with different stoichiometry, and this difference in binding stoichiometry was taken into account when calculating the affinity constants $-K_{\text {assoc }}$ (Table 2 ). These results show only a two- to threefold difference in affinity to RNA between the PARP1 proteins - with PARP1-FL having the highest affinity, whereas $\Delta \mathrm{Zn} 3$ showed the lowest affinity, followed by $\Delta \mathrm{Zn} 1 \Delta \mathrm{Zn} 2$ (Table 2). These data are in line with previous studies that showed that PARP1 binds 

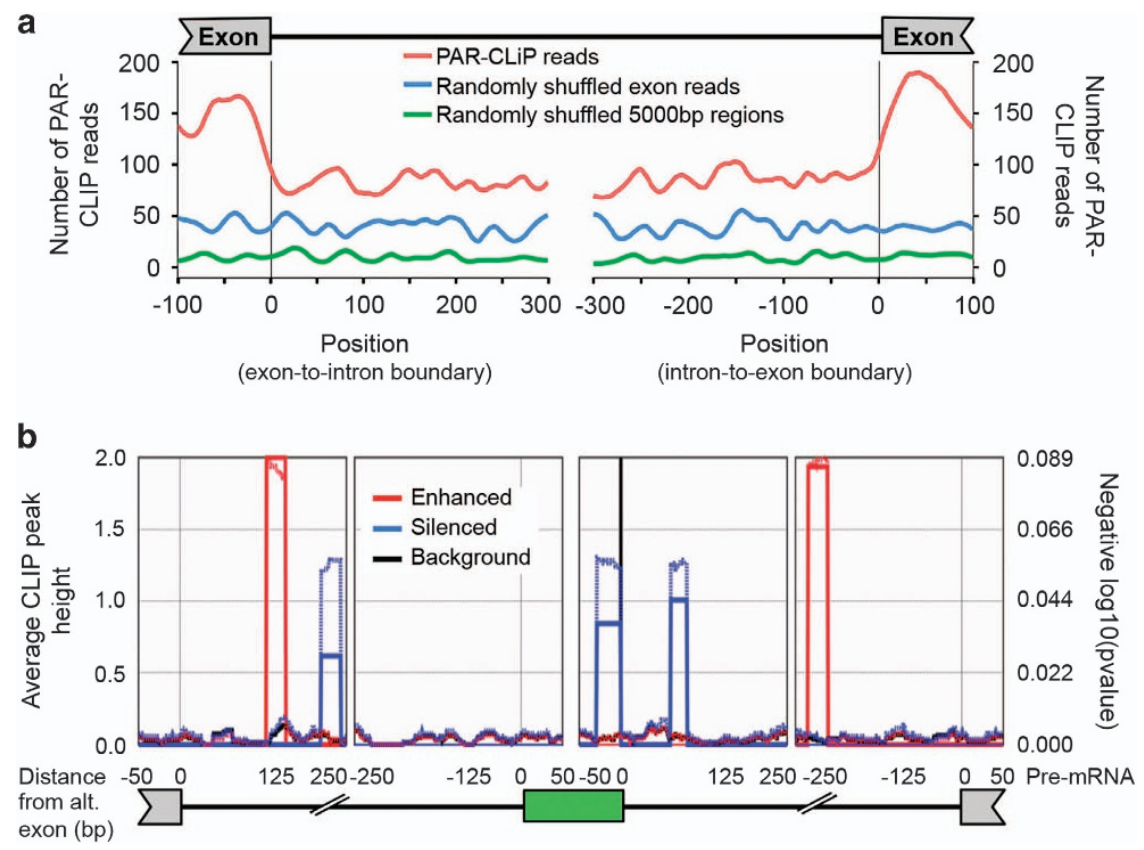

Figure 5 Positional analysis of PARP1-PAR-CLIP tags, with respect to splicing functions. (a) The number of PAR-CLIP sequences covering exon boundaries (red) compared to the number of sequences covering randomly shuffled exons (blue) and randomly selected 5 000-bp regions (green). (b) Maps for PARP1-PAR-CLIP read enrichment for the skipped exon events from RNA-seq of PARP1 knockdown and control (non-targeting siRNA) experiments are shown for enhanced (red) and silenced (blue) splicing events. Solid lines represent the peak quality score (peak height) as scaled on the left. Dotted lines represent the significance score ( $P$-value) as scaled on the right. The level of significance was determined by comparison to a 'Background set' of 32114 of non-impacted alternative exons (rMATs FDR $>50 \%$ ) in expressed genes (FPKM $>5.0$ ). Only events showing $P \leq 0.05$, FDR $\leq 0.05$, and a minimum inclusion level difference $\geq 0.1$ were considered. The green box indicates the PARP1regulated exon.

RNA via its zinc-finger 3 domain [34]. Interestingly, deletion of another region previously implicated in binding RNA (WGR) did not significantly change the affinity from that of the PARP1-FL. Similar binding affinity results were obtained using RNAs of different lengths (20 and 24 nt; Supplementary Table S6). At first surprising, similar small differences in affinity have also been recorded for the binding of these constructs to DNA [35], although PARP1 is a well-known DNAbinding protein. These previous results hypothesized that all the domains of PARP1 contribute to its DNAbinding interactions. We believe that a similar scenario is occurring with PARP1 binding to RNA.

Following on these results, we examined the possibility that RNA activates PARP1 and showed that, just like DNA, RNA activates PARP1, albeit at a lower extent (Supplementary Figure S10). Finally, we performed a competition assay to test whether PARP1 preferentially binds DNA to RNA. Equal concentration of radiolabeled 19-mer RNA and radiolabeled ssDNA of the same sequence was incubated together with increasing concentrations of the different PARP1 constructs. As the ssRNA and ssDNA of the same sequence run with different gel mobility, it allowed us to quantify the disappearance of the RNA and DNA in the presence of these recombinant PARP1 proteins. This analysis revealed that PARP1-FL had a 25-fold affinity to DNA than RNA (Figure 8a for PARP1-FL). A similar result was observed with the other constructs (Table 3) except for the $\Delta \mathrm{Zn} 1 \Delta \mathrm{Zn} 2$ mutant. This mutant switched PARP1's binding preference from DNA to RNA, with a sevenfold preference for RNA to DNA (Figure $8 \mathrm{~b}$ and Table 3). These results indicate that, once the $\mathrm{Zn} 1 \mathrm{Zn} 2$ site is unavailable, PARP1 preferentially binds RNA and suggest that the DNA binding is different from the site needed to bind RNA.

\section{Discussion}

The transcriptome analysis performed here by highthroughput PAR-CLIP sequencing provides new insights into the endogenous RNA targets of $P A R P 1$. We found that PARP1 binds RNA in vivo (Figure 1). 
a
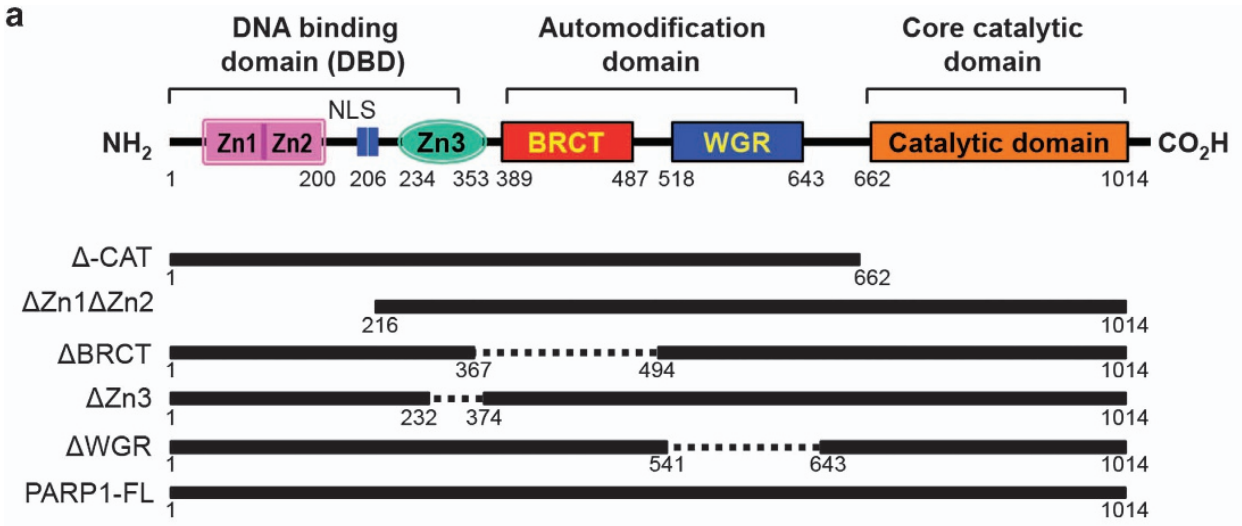

b

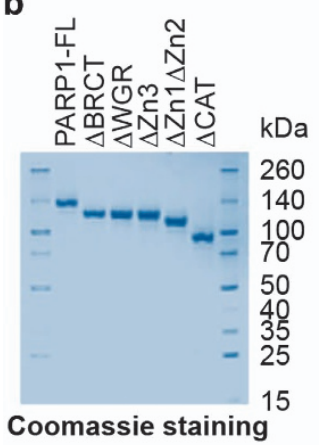

C

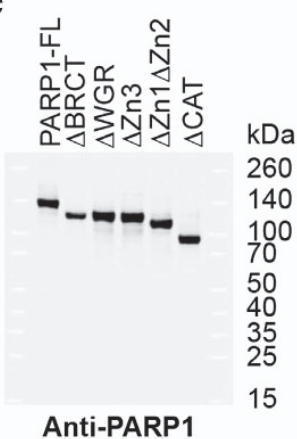

d

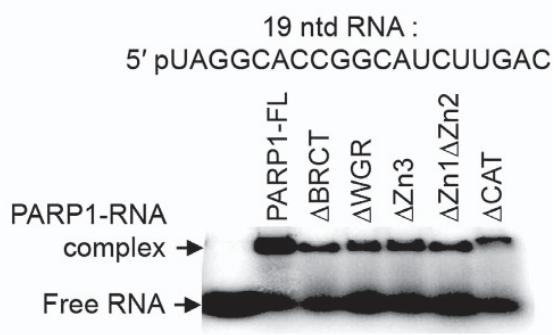

19 ntd RNA :

-terminal antibody

Figure 6 PARP1-RNA domain interactions. (a) Schematic structure of PARP1 showing the various functional domains important in PARP1 activation, localization, and activity. The first three N-terminal domains (residues 1-353) are zinc-finger DNA-binding domains with distinct functions in PARP1 DNA nick-mediated activation. The central, auto-modification region of PARP1 contains a BRCT domain (residues 389-487), as well as flanking residues that serve as sites of auto-ADP ribosylation. Adjacent to the BRCT domain is a WGR domain (residues 518-643) followed by the catalytic domain (residues 662-1 014), which possesses activities related to the ADP-ribose adduct formation, elongation, and branching activity. Below are the various PARP1 constructs created to test the RNA-binding activity of PARP1. (b) Coomassie gel staining of purified recombinant full-length PARP1 and the truncated PARP1 proteins. (c) Western blot analysis of PARP1 and truncated PARP1 proteins. (d) Gel shift assays showing the binding of RNA by various proteins, PARP1 full-length, and truncations.

We also observed that, whereas the main target of PARP1-RNA binding in vivo is mRNA, it also binds other non-coding RNAs (Figure 2), suggestive of a functional role of PARP1 in their regulation. Within mRNAs, we find that PARP1 associates mainly with intronic sequences (Figure 2). However, since introns are very long and PARP1-RNA targets could target different regions of a particular intron, we also analyzed the density of the reads at functional splice sites. Our results show that there is a high density of PARP1RNA binding at exon-intron boundaries and intronexon boundaries (Figure 5). These results could suggest that PARP1 demarcates exons. Interestingly, we previously had showed that PARP1 binds GC-rich nucleosomes at exon boundaries [20]. It is therefore logical to assume that it binds to similar regions on chromatin as well as on RNA, possibly by recognizing specific sequences or structures on DNA and/or RNA. However, additional studies are needed to determine the structural implications of PARP1 binding. We further combined the PAR-CLIP-seq analysis with full transcriptome-wide analysis of gene expression and splicing changes upon PARP1 depletion. Combining PAR-CLIP and RNA-seq data allowed us to draw a PARP1 RNA map, which suggested that the binding of PARP1 on exons and in intronic regions immediately surrounding the regulated skipped exon leads to silencing of the downstream exon. PARP1 binding to introns further upstream and downstream of the skipped exon enhances exon inclusion (Figure 5b). The high distribution of PARP1 in introns (Figure 2) enhances the idea of a regulatory role of PARP1 in splicing, as intronic-binding proteins such as HNRNPU [36], HNRNPH1 [37], and HUR [38] have 

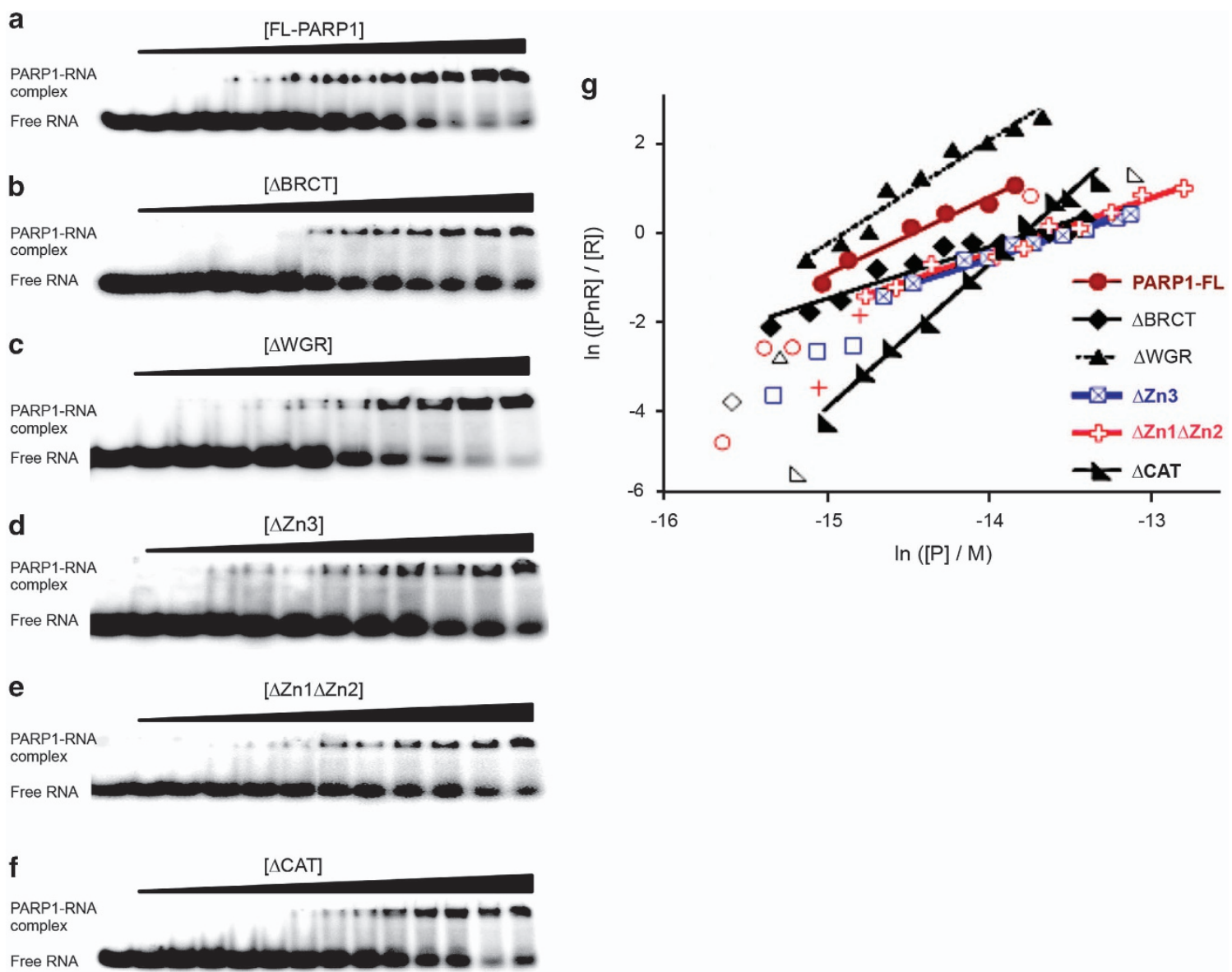

Figure $7 \mathrm{Gel-shift} \mathrm{assays} \mathrm{for} \mathrm{the} \mathrm{binding} \mathrm{of} \mathrm{PARP1} \mathrm{proteins} \mathrm{to} \mathrm{single-stranded} \mathrm{19-mer} \mathrm{RNA.} \mathrm{(a-f)} \mathrm{Full-length} \mathrm{PARP1} \mathrm{proteins} \mathrm{and}$ the truncated mutants bind single-stranded 19-mer RNA. In these experiments, RNA (0.05 $\mu \mathrm{m})$ was incubated with increasing concentrations of the six different [PARPs] (0-2.5 $\mu \mathrm{m}$ from left to right). The lower bands indicate free RNA and the upper bands are PARP1-RNA protein complexes. (g) Associated binding isotherm analysis. The slope of each isotherm is a measure of stoichiometry for the individual PARP1 protein binding to the RNA. Values of $n$ and $K$ are summarized in Table 2.

been implicated in splicing decisions. Under this scenario, the binding of PARP1 to intronic sequences mediates splicing; however, it can also remain associated with the mature mRNAs to help in other posttranscriptional mRNA processes. This seems to be occurring, as we observe a high PARP1 PAR-CLIP read density, at the ends of exons (exon-intron and intron-exon boundaries depicting $3^{\prime}$ and $5^{\prime}$ splice sites, respectively), and is in line with other intron-binding proteins [39]. Noteworthy is the fact that proteins that bind at exons interact with the RNA after transcription and initial RNA processing, whereas the intron binders are present during transcription [21], thus supporting their role in co-transcriptional splicing. However, because of the low CLIP efficiency (only $\sim 1 \%$ of transcripts are crosslinked), it is difficult to distinguish whether the PARP1-RNA interactions are on pre-
mRNA transcripts or whether a subset of these $m R N A s$ is subsequently processed (in either alternative exons or poly (A) sites). On the other hand, our RNA map of PARP1 binding (Figure 5b) provides a functional landscape of significantly skipped alternative splicing regulation by PARP1 that can be used in future studies to further characterize the regulation of AS by PARP1. PARP1 could be modulating splicing decisions through two mutually non-exclusive mechanisms: (i) maintaining a chromatin structure that affects RNA polymerase kinetics and/or (ii) recruiting and PARylating splicing factors to splice sites on nascent mRNAs while bound onto chromatin.

PARP1 has been implicated in many cellular processes. In this study, we focused on the observation that PARP1 is involved in splicing regulation [20]. The means by which PARP1 regulates alternative splicing is 
Table 2 Stoichiometries $(n)$ and association constants $\left(K_{\text {assoc }}\right)$ for PARP1-FL and truncation mutants to 19-mer ssRNA

\begin{tabular}{lcl}
\hline Protein type & $K_{\text {assoc }}\left(\mathrm{M}^{-1}\right)$ & Stoichiometry $(n)$ \\
\hline PARP1-FL & $(1.95 \pm 0.08) \times 10^{6}$ & $1.75 \pm 0.20$ \\
PARP1- $\Delta$ WGR & $(2.91 \pm 0.15) \times 10^{6}$ & $2.33 \pm 0.18$ \\
PARP1- $\Delta$ CAT & $(1.05 \pm 0.07) \times 10^{6}$ & $3.31 \pm 0.32$ \\
PARP1- $\Delta$ BRCT & $(0.97 \pm 0.06) \times 10^{6}$ & $1.17 \pm 0.10$ \\
PARP1- $\Delta$ Zn1 $\Delta Z n 2$ & $(0.86 \pm 0.04) \times 10^{6}$ & $1.26 \pm 0.11$ \\
PARP1- $\Delta Z n 3$ & $(0.78 \pm 0.03) \times 10^{6}$ & $1.19 \pm 0.08$ \\
\hline
\end{tabular}

still unknown. Earlier understanding of gene expression regulation suggested that DNA-binding proteins responded to sequence composition and chromatin context to promote transcription of RNA [40, 41]. RNA-binding proteins (RBPs) then bind these nascent transcripts to direct mRNA splicing, stability, localization, and translation [42, 43]. However, recent advances profiling nucleic acid-protein interactions find that many DNA-binding proteins also associate with RNA to modulate both transcriptional and posttranscriptional outcomes [19, 44-46], blurring this long-standing dogma for gene regulation. The results presented here also find that PARP1, a well-known DNA/chromatin-binding factor, binds RNA, adding to this growing list of proteins interacting with both DNA and RNA to affect gene regulation. Our study further suggests that PARP1 binding to RNA may regulate gene splicing and/or generally different levels of RNA biogenesis. Collectively, these studies suggest a more intertwined gene regulatory network (transcription and splicing) than had been previously appreciated.

Indeed, it is now known that splicing is tightly integrated with gene expression [47, 48], with splicing controlling gene expression via nonsense-mediated [49] or spliceosome-mediated [50] decay pathways. Unspliced and partially spliced transcripts can be deleterious for the cell [51, 52] and several qualitycontrol pathways exist to degrade these faulty transcripts. The first and main line of protection (degradation of these faulty transcripts) is through the nuclear exosome process [53-55]. If this fails, a second line of defense occurs via cytoplasmic surveillance pathways [52], leading to cytoplasmic degradation. This can be triggered in two ways - the nonsense-mediated decay (NMD) pathway that recognizes premature stop codons $[56,57]$ or by the non-stop decay pathway that identifies transcripts lacking stop codons [58]. Interestingly, PARP1 depletion led to an upregulation in the expression of transcripts for protein products involved in the NMD pathway, and a decrease in transcripts of proteins involved in poly-A-RNA binding, showing a clear intersection of PARP1 in RNA biogenesis. Several studies implicate PARP1 in several steps of RNA biogenesis such as RNA metabolism [59], mRNA metabolism, and protein synthesis [3, 60]. Furthermore, splicing factor $3 \mathrm{~A}$ subunit 1 , splicing factor $3 \mathrm{~B}$ subunit 1, splicing factor $3 \mathrm{~B}$ subunit 2 [61], and alternative-splicing factor $1 /$ splicing factor 3 [62] are either targets of poly(ADP-ribosyl)ation or bind directly to PARP1. The function of poly (ADP-ribose) binding, the binding to PARPs, and ADP ribosylation of these splicing factors is not well understood.

In these studies, we show that PARP1, a known DNA-binding protein, binds RNA both in vivo (Figure 1 and Supplementary Figure S1) and in vitro (Figure 7). Our forward competition assays of PARP1 binding to DNA and RNA showed that, in the absence of the $\mathrm{Zn} 1 \mathrm{Zn} 2$ domain, PARP1 preferred binding to RNA than to DNA (Figure 8). These results are consistent with our idea of PARP1's role in cotranscriptional splicing [20], where PARP1 binds to chromatin using the $\mathrm{Zn} 1 \mathrm{Zn} 2$ domain, and when that site is used it still has the ability to bind to nascent mRNA through another domain. Does PARP1 recognize a specific RNA motif? Previous studies showed that PARP1 binds the DNA motif, AGGCC [63], and/or binds to the vicinity of the DNA motif, GGAAGG [64]. In our analysis, we failed to find an enriched RNA motif for PARP1 binding; we did, however, find that PARP1 binds to RNA sequences enriched in GC-rich sequences (Figure 3 and Supplementary Figure S6). It is tempting to speculate that in binding to these GC-rich sequences PARP1 recognizes a structure formed by these sequences. One such structure is formed by G-quadruplexes, which have also been implicated in splicing regulation. In fact, PARP1 binds G-quadruplexes in vivo [65-67]. However, additional studies will be needed to test whether PARP1 RNA targets form structures such as the G-quadruplex.

Our results showing that deletion of the third zinc finger of PARP1 resulted in the lowest affinity of this mutant protein for RNA (Figure 7 and Table 2) support the idea that PARP1 uses its Zn3 to bind RNA in vitro [34] or pRNA [17]. The small difference in affinity between PARP1-FL and its truncation mutants could imply that either: (i) all regions contribute to RNA binding or (ii) as yet, there is an undiscovered RNA-binding region of PARP1. These possibilities are not far-fetched since other PARPs lacking of some of the domains of PARP1 bind to RNA. For instance, PARP12 and PARP13 bind RNA through its zinc 


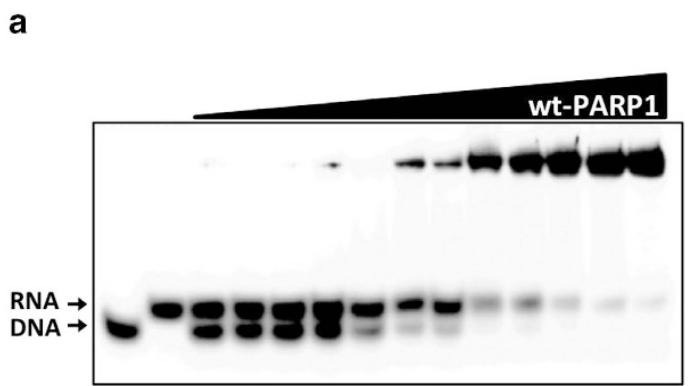

b

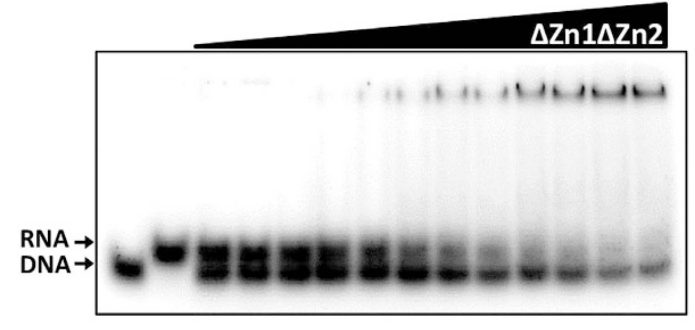

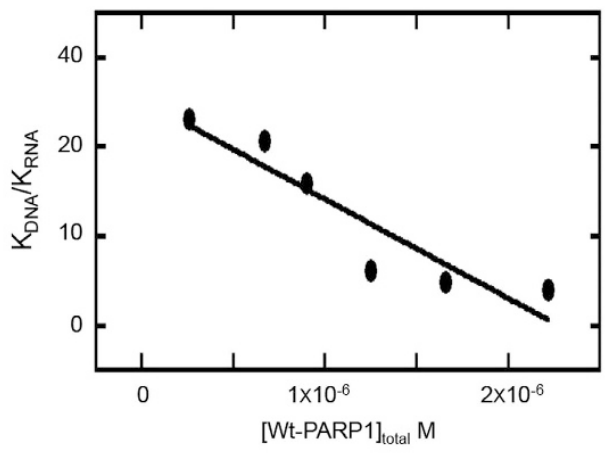

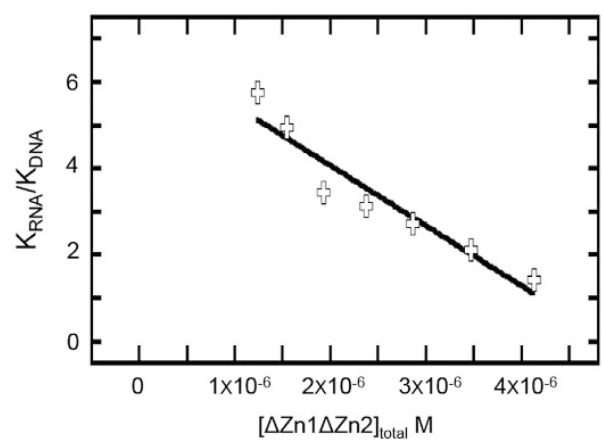

Figure 8 In the absence of Zn1Zn2, PARP1 preferentially binds RNA in vitro. PARP1 was incubated with radiolabeled ssRNA and radiolabeled ssDNA of the same sequence in the same reaction. (a) Radiolabeled 19-ntd ssRNA and ssDNA were incubated with increasing concentrations of PARP1-FL. Left, EMSA of PARP1-DNA and PARP1-RNA complex formation with increasing concentrations of PARP1-FL. The DNA band disappears faster than the RNA band. Right, graph depicts the relationship between radiolabeled DNA/RNA and increasing concentration of recombinant PARP1 in the same binding reaction. (b) Radiolabeled 19ntd ssRNA and ssDNA were incubated with increasing concentrations of $\Delta \mathrm{Zn} 1 \Delta \mathrm{Zn} 2$. Left: EMSA analyses showing the formation of $\Delta \mathrm{Zn} 1 \Delta \mathrm{Zn} 2-\mathrm{DNA}$ and $\Delta \mathrm{Zn} 1 \Delta \mathrm{Zn} 2-\mathrm{RNA}$ complexes with increasing concentrations of $\Delta \mathrm{Zn} 1 \Delta \mathrm{Zn} 2$. In the case of $\Delta \mathrm{Zn} 1 \Delta \mathrm{Zn} 2$, the RNA band disappears faster than the DNA. Right, graph depicts the relationship between radiolabeled DNA/RNA and increasing concentration of recombinant $\Delta \mathrm{Zn} 1 \Delta \mathrm{Zn} 2$ in the same binding reaction. Because of the inability to differentiate PARP1-DNA complexes from PARP1-RNA complexes on the gel, the rate of disappearance of the DNA and RNA bands was used as a proxy to measure the affinity of PARP1 for either DNA or RNA.

fingers, whereas PARP14 and PARP10 have possible RRMs present on different protein domains [68]. In addition, PARP7, which lacks these zinc-finger domains, still binds RNA [69]. As of now, it is not clear whether there is an RNA recognition motif on PARP1, although in addition to the zinc-finger 3 domain the WGR domain can also bind RNA [34]. Future studies will be critical to determine the exact RNA recognition motif of PARP1.

In light of PARP1's in vivo binding to RNA, its effect on splicing, and its importance in the regulation of transcript expression of some of the proteins important for NMD and poly-A binding, it is provocative and highly suggestive to hypothesize that PARP1 is a protein involved in genome surveillance. This hypothesis seems plausible if one considers its role in DNA repair; whereas PARP1 does not execute the repair itself, it binds to the site of damage and recruits repair proteins to the site of repair [1]. Furthermore, in transcription regulation, it stalls polymerase elongation $[6,70]$, thereby possibly allowing proper genome surveillance. Once surveillance is complete, in the absence of any DNA damage, it then PARylates histones, releasing the repression on polymerase elongation [5, 6]. We believe that this is also a likely scenario in splicing. PARP1 by itself does not splice, but binds to specific splice sites [20] (Figure 5), possibly recruiting/ activating splice factors to that region. Although recruitment of splice factors has not been shown, PARP1 PARylates and activates splicing factors [62]. In addition, this idea is also further bolstered when one considers its functions at the $3^{\prime}$ ends of mRNA where PARP1 PARylates poly-A binding protein (PAP), thus decreasing the ability of the modified PAP to bind 
Table 3 Relative binding affinities from competition EMSA experiments for PARP1-FL and truncation mutants to 19-mer ssDNA compared to 19-mer ssRNA

\begin{tabular}{lcc}
\hline Protein type & $K_{\mathrm{DNA}} / K_{\mathrm{RNA}}$ & $K_{\mathrm{RNA}} / K_{\mathrm{DNA}}$ \\
\hline PARP1-FL & $25.3 \pm 3.0$ & $0.001 \pm 0.02$ \\
PARP1- $\Delta$ WGR & $26.5 \pm 2.4$ & $0.024 \pm 0.011$ \\
PARP1- $\Delta$ CAT & $15.7 \pm 3.9$ & $0.046 \pm 0.021$ \\
PARP1- $\Delta$ BRCT & $42.3 \pm 1.2$ & $0.001 \pm 0.016$ \\
PARP1- $\Delta \mathrm{Zn} 1 \Delta \mathrm{Zn} 2$ & $0.0153 \pm 0.021$ & $7.2 \pm 0.7$ \\
PARP1- $\Delta \mathrm{Zn} 3$ & $23.1 \pm 5.2$ & $0.032 \pm 0.017$ \\
\hline
\end{tabular}

RNA. This PARylation effect has also been shown for several other $3^{\prime}$ processing factors such as PABPN1 and all CPSF subunits [59], pointing to the possibility that PARP1 might be a general regulator of $3^{\prime}$ processing. Lastly, the study of PARP1 under different scenarios has probably led to the idea that it acts in so many functions; however, it is also tempting to speculate that it acts generally as surveillance molecule that ensures genome stability.

Our understanding of the role of PARPs and PAR in transcriptional and post-transcriptional regulation of gene expression through modulation of RNA is still in its early stages. Our studies, however, provide a very useful platform to begin to tease, uncover, and decipher PARP1's role in the many steps in RNA biogenesis.

\section{Materials and Methods}

\section{Cell culture}

HeLa cells were used for PAR-CLIP experiments. Cells were grown at $37^{\circ} \mathrm{C}$ in a humidified environment containing $5 \% \mathrm{CO}_{2}$ and 95\% air in Dulbecco's modified Eagle's medium (Sigma) containing $1 \mathrm{~mm}$ sodium pyruvate, $0.1 \mathrm{~mm}$ nonessential amino acids, and supplemented with $10 \%$ fetal bovine serum, $100 \mathrm{U} \mathrm{ml}^{-1}$ penicillin, and $100 \mu \mathrm{g} / \mathrm{ml}$ streptomycin. For each experiment, $\sim 6 \times 10^{8}$ cells $(\sim 60 \times 15 \mathrm{~cm}$ cell culture plates $)$ were used.

\section{PAR-CLIP methodology}

Cells were cultured to $80-90 \%$ confluency, and then treated overnight with 4-thiouridine to a final concentration of $100 \mu \mathrm{M}$ added directly to the cell culture medium. Cells were washed with ice-cold phosphate-buffered saline (PBS), the liquid was aspirated and the plates placed over ice and then irradiated with UV light at $365 \mathrm{~nm}\left(150 \mathrm{~mJ} \mathrm{~cm}^{-2}\right)$. Cells were then scraped off the plates and collected by centrifuging at 2000 r.p.m. for $10 \mathrm{~min}$.

PAR-CLIP was performed as previously described [26] with some modifications. Briefly, $10 \mathrm{ml}$ of packed cell pellet-UVtreated cells were lysed with 3 volumes of $1 \times$ NP40 lysis buffer on ice for $5 \mathrm{~min}$. Cells were pelleted by centrifugation at $18000 \mathrm{~g}$ for 15 min using an Eppendorf 5810R centrifuge 5810R with an
A-4-81swing bucket rotor. The supernatant was filtered using a $5 \mu \mathrm{M}$ syringe filter (Sterile Acrodisc Syringe Filters with Supor Membrane; Ann Arbor, MI 48103 USA) to remove cellular debris. The filtrate was partially treated with RNase T1 (Roche,

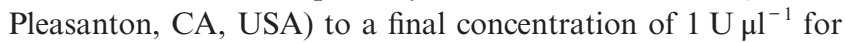
$15 \mathrm{~min}$. The RNase-treated supernatant was then incubated for $2 \mathrm{~h}$ with $600 \mu \mathrm{l}$ of protein A dynabeads (Invitrogen, Thermo fisher Scientific, Waltham, MA,USA) bound to $15 \mu \mathrm{g}$ of antiPARP1 antibody (Active Motif, Carlsbad, CA, USA) or control IgG antibody. The beads were washed three times and the immunoprecipitated RNA was digested again with RNase T1 to

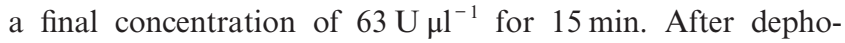
sphorylation, the RNA segments crosslinked to PARP1 were 5'radiolabeled using $\gamma{ }^{32} \mathrm{P}-\mathrm{ATP}$ and $\mathrm{T} 4$ polynucleotide kinase (Promega Madison, WI, USA) in one original bead volume. After several washes, each CLIP sample (on the beads) was then treated with $5 \mathrm{U}$ of DNase1 (NEB Ipswich, MA, USA) for every $100 \mu \mathrm{l}$ of bead volume for $15 \mathrm{~min}$ at $37^{\circ} \mathrm{C}$. DNasel was inactivated by adding $5 \mathrm{mM}$ EDTA and heated at $65^{\circ} \mathrm{C}$ for $10 \mathrm{~min}$. Samples were then resuspended in SDS-PAGE loading buffer, incubated at $95^{\circ} \mathrm{C}$ for $5 \mathrm{~min}$ to denature, and the PARP1-RNA crosslinks were release. The samples were then separated on 4 $12 \%$ NuPAGE gels (Invitrogen) and transferred onto nitrocellulose membranes (1/10th of the sample was used for immunoblotting and the rest of the sample was used for autoradiography). The gel containing 1/10th of the sample and the membrane containing 9/10th of the sample were exposed to a phosphorimager screen overnight and visualized by scanning on a Typhoon FLA 9500. PARP1-RNA complexes were cut from the membrane, treated with proteinase $\mathrm{K}$ (Roche), followed by Phenol/Chloroform/IAA extractions and ethanol precipitation. The recovered RNA was used for cDNA library preparation.

For this purpose we used NEBNext Multiplex Small RNA Library Prep Set for Illumina (Set 1). Library preparation including $3^{\prime}$ and $5^{\prime}$ SR Adaptor ligations, reverse transcription, and PCR amplification, which were performed according to the manufacturer's protocol. To remove adaptor-only ligation products, after every step of the protocol ( $3^{\prime}$ adapter ligation and $5^{\prime}$ adapter ligation) samples were purified using $15 \%$ acrylamide8M Urea gels. Lastly, after limited PCR amplification PCR products were size-selected on a 3.5\% NuSieve (Lonza Walkersville MD, USA) low-melting point agarose gel. Expectant PCR products were eluted using the 'crush and soak' method, followed by purification using a Qiagen min-elute PCR column. Samples were then first cloned into the Topo TA vector for pilot analysis and then sequenced using $100 \mathrm{bp}$ paired-end sequencing on an Illumina HiSeq 2500.

\section{Western blot analysis}

Protein samples were resuspended in SDS sample buffer, and then separated on 4-12\% NuPAGE gel (Invitrogen, Thermo fisher Scientific, Waltham, MA, USA), transferred onto nitrocellulose membranes, blocked with $5 \%$ fat-free milk in PBST, and incubated with primary antibodies for $16 \mathrm{~h}$ at $4{ }^{\circ} \mathrm{C}$. After several washes with PBST, the membranes were incubated with secondary antibodies conjugated to alkaline phosphatase for $1 \mathrm{~h}$ at room temperature, and a signal was developed with ECL 
reagents (GE Healthcare, Pittsburg, PA, USA). Images were obtained using a Typhoon 9400.

Antibodies. The following antibodies were used in this study: CHIP-grade PARP1 antibody (Active Motif: 39559).

PARP1 knockdown. The ON-TARGETplus Human PARP1 siRNAs (purchased from GE Healthcare Dharmacon, Dharmacon, Lafayette, CO, USA) and DharmaFECT 1 transfection reagent were used to deliver siRNAs into HeLa cells according to the manufacturer's protocol. In brief, $2 \times 10^{5}$ cells/well plated on six-well plates were starved by incubating the cells in $2 \mathrm{ml}$ of antibiotic-free complete medium with $10 \%$ serum for $2 \mathrm{~h}$. Then, $50 \mathrm{nM}$ of the ON-TARGETplus Human PARP1 siRNAs were added to serum-free DMEM $(180 \mu \mathrm{l})$ in one tube, and DharmaFECT $1(2.4 \mu \mathrm{l})$ was added to $197.6 \mu \mathrm{l}$ of serum-free medium in another tube. The contents of each tube were gently mixed individually for $5 \mathrm{~min}$ at room temperature and then combined. This mixture was then incubated at room temperature for an additional $20 \mathrm{~min}$. For control samples $2.4 \mu \mathrm{l}$ of DharmaFECT 1 was added to $400 \mu \mathrm{l}$ serum-free medium. Subsequently, an additional $1600 \mu 1$ serum-free medium was added to each mixture of $400 \mu \mathrm{l}$ for a final volume of $2000 \mu \mathrm{l}$ transfection medium and a final siRNA concentration of $50 \mathrm{mM}$. The starvation media from the cells were then removed and replaced with the $2000 \mu \mathrm{l}$ transfection mixture. Cells were further incubated for $1 \mathrm{~h}$ at $37^{\circ} \mathrm{C}$ before $10 \%$ serum was added. Cells were then allowed to grow for $24-48 \mathrm{~h}$. This procedure was repeated three times, every $24-48 \mathrm{~h}$. After that, the cells were collected and analyzed for mRNA or PARP1 protein to check the target gene knockdown efficiency. A total of seven independent experiments were performed.

Purification of human full-length PARP1 and its truncated mutants. His-tagged PARP1 expression vectors were a kind gift from the Pascal Laboratory (University of Montreal), and purified as previously described [71]. Briefly, the sequences corresponding to full-length PARP1 (aa 1-1 014), $\triangle \mathrm{CAT}$ (aa 1662), $\Delta \mathrm{Zn} 1 \Delta \mathrm{Zn} 2$ (aa 216-1 014), $\Delta \mathrm{Zn} 3$ (truncated aa: 232-374), $\triangle B R C T$ (truncated aa: 367-494), and $\triangle$ WGR (truncated aa: 518-654) were cloned into pET28 expression vector. Proteins were expressed in One Shot BL21 (DE3) pLysS competent cells (E. coli) and purified using three subsequent chromatographic fractionations: (1) a $\mathrm{Ni}^{2+}$ affinity column (Ni-NTA agarose, Qiagen Valencia, CA, USA), (2) a heparin column (5-ml HiTrap Heparin HP Column, GE Healthcare), and (3) a gel filtration column (Superdex S200 size exclusion column, GE Healthcare, Pittsburg, PA, USA). Pooled fractions were required to monitor expression, purity and analyze fractions; we used SDS-PAGE (NuPage, 4-12\% Bis-Tris, Invitrogen). The desired fractions were then concentrated using an Amicon Ultra spin concentrator with a 10000 molecular weight cutoff (Millipore, Billerica, MA 01821 USA). Protein concentrations were determined with the Pierce BCA Protein Assay Kit (Thermo Scientific, Thermo fisher Scientific) and by absorbance at $280 \mathrm{~nm}$ using the molar extinction coefficients calculated for each PARP1-protein: $1.19 \times 10^{5} \mathrm{M}^{-1} \mathrm{~cm}^{-1}$ (PARP1-FL), $1.14 \times 10^{5}$ $\mathrm{M}^{-1} \mathrm{~cm}^{-1}$ ( $\left.\triangle \mathrm{BRCT}\right), 9.23 \times 10^{4} \mathrm{M}^{-1} \mathrm{~cm}^{-1}(\Delta \mathrm{WGR}), 9.82 \times 10^{4}$
$\mathrm{M}^{-1} \mathrm{~cm}^{-1}(\Delta \mathrm{Zn} 3), \quad 8.82 \times 10^{4} \quad \mathrm{M}^{-1} \mathrm{~cm}^{-1} \quad(\Delta \mathrm{Zn} 1 \Delta \mathrm{Zn} 2), \quad$ and $8.43 \times 10^{4} \mathrm{M}^{-1} \mathrm{~cm}^{-1}(\Delta \mathrm{CAT})$.

\section{Electrophoretic mobility-shift assay}

Electrophoretic mobility-shift assay (EMSA) analysis was performed according to standard procedures. RNA oligonucleotides (19-mer with the sequence: CGUACGCGGG UUUAAACGA) containing the binding sites for PARP1 were labeled at the $5^{\prime}$ termini with ${ }^{32} \mathrm{P}$. For binding assays, a constant amount $(0.05 \mu \mathrm{M})$ of labeled RNA probe was preincubated with increasing concentrations of PARP1 protein range $0-2.5 \mu \mathrm{M}$ in a final volume of $20 \mu \mathrm{l}$ at room temperature for $30 \mathrm{~min}$ in $25 \mathrm{mM}$ Tris ( $\mathrm{pH} 7.5$ ), $75 \mathrm{mM} \mathrm{NaCl}, 50 \mathrm{mM}$ arginine, $0.1 \mathrm{mM}$ TCEP, and $0.1 \mu \mathrm{g} \mu \mathrm{l}^{-1}$ bovine serum albumin. The RNA-protein complexes were then analyzed by electrophoresis on native $10 \%$ polyacrylamide gels (75:1 acrylamide:bisacrylamide) in Trisborate-EDTA buffer, followed by autoradiography. Autoradiographic images were captured on a storage phosphor screens (type GP, GE Healthcare, Pittsburg, PA, USA) detected with a Typhoon FLA 9500 and quantitated with Image-Quant TL software (GE Healthcare).

Self-consistent estimates of binding stoichiometry $(n)$ and the association constant $\left(K_{n}\right)$ were obtained by the method of Fried and Crothers [72, 73]. For a single binding step in which $n$ protein molecules associate with RNA ( $\mathrm{R}$ is used to represent RNA in the equation) the association constant is $K_{n}=\left[\mathrm{P}_{n} \mathrm{R}\right] /$ $[\mathrm{R}][\mathrm{P}]^{n}$ free. Separating variables and taking logarithms gives:

$$
\ln \left(\left[\mathrm{P}_{\mathbf{n}} \mathrm{R}\right] /[\mathrm{R}]\right)=\operatorname{nln}[\mathrm{P}]_{\text {free }}+\ln \mathrm{K}_{\mathbf{n}}
$$

For these experiments, $[\mathrm{R}]_{\text {total }} \ll[\mathrm{P}]_{\text {total }}$, so $[\mathrm{P}]_{\text {total }}$ is an acceptable estimate of $[\mathrm{P}]_{\text {free }}$. Thus, a graph of $\ln \left(\left[\mathrm{P}_{n} \mathrm{R}\right] /[\mathrm{R})\right.$ as a function of $\ln [\mathrm{P}]_{\text {free }}$ has a slope equal to the stoichiometry of the binding step, $n$. The equilibrium constant is most simply estimated at the midpoint, where $\ln \left(\left[\mathrm{P}_{n} \mathrm{R}\right] /[\mathrm{R}]\right)=0$ and $\ln \mathrm{K}_{n}=-n$ $\ln [\mathrm{P}]_{\text {free. }}$. Because the assessed stoichiometries differ for different complexes, we estimated the equilibrium constants for the overall reactions, $\mathrm{K}_{n}\left(\mathrm{M}^{-n}\right)$ and the corresponding monomerequivalent association constants, $K\left(\mathrm{M}^{-1}\right)$.

For competition assays, equimolar amounts $(0.05 \mu \mathrm{M})$ of radiolabeled ssRNA and radiolabeled ssDNA of the same nucleotide sequence were mixed in a $20 \mu$ reaction and incubated with increasing concentrations of PARP1-FL or its truncated mutants $(0-2.5 \mu \mathrm{M})$. The binding reaction was performed as described above. The ratio of binding affinities $K_{\mathrm{DNA}} / K_{\mathrm{RNA}}$ was determined from the relationship [72, 73]

$$
\mathrm{K}_{\text {DNA }} / \mathrm{K}_{\text {RNA }}=\left(\left[\mathrm{P}_{\mathbf{m}} \mathrm{D}\right] /[\mathrm{D}][\mathrm{P}]^{\mathbf{m}}\right) /\left(\left[\mathrm{P}_{\mathbf{n}} \mathrm{R}\right] /[\mathrm{R}][\mathrm{P}]^{\mathbf{n}}\right)
$$

Where $m$ and $n$ are the stoichiometries of the binding of protein to DNA and RNA, correspondingly, calculated from single titration experiments of PARP1 proteins (PARP1-FL and mutants) to DNA and RNA (PARP1-DNA titration is not shown). As PARP1-RNA and PARP1-DNA complexes comigrate under the electrophoretic conditions of our experiments, $\left[\mathrm{P}_{m} \mathrm{D}\right]$ and $\left[\mathrm{P}_{n} \mathrm{R}\right]$ were calculated according to the relationships $\left[\mathrm{P}_{m} \mathrm{D}\right]=\left([\mathrm{D}]_{0}-[\mathrm{D}]_{\text {free }}\right) \times m$ and $\left[\mathrm{P}_{n} \mathrm{R}\right]=\left([\mathrm{R}]_{0}-[\mathrm{R}]_{\text {free }}\right) \times n . \quad[\mathrm{D}]_{0}$ and $[R]_{0}$ are the initial concentrations of DNA and RNA, respectively, and $[\mathrm{D}]_{\text {free }}$ and $[\mathrm{R}]_{\text {free }}$ are the free concentrations of the competitors at each PARP1 concentration in the initial 
titration experiment. Because all the components of the equation can either be measured or calculated from our experimental data, $K_{\mathrm{DNA}} / K_{\mathrm{RNA}}$ for the modest values of [PARP1] was calculated from the plots and the ratio of binding affinities estimated from the linear part of the plot by extrapolation to the $[\mathrm{PARP} 1]=0$.

\section{RNA markers used in PAR-CLIP experiments. 19-mer: CCG UACGCGGGUUUAAACGA 24-mer: CGUACGCGGA AUAGUUUAAACUGU}

\section{ssRNA used for gel-shift. 19-mer: UAGGCACCGGCAUC UUGAC 20-mer: CCGUACGCGGGUUUAAACGA 24-mer: CGUACGCGGAAUAGUUUAAACUGU}

\section{ssDNA used for gel-shift. 19-mer: TAGGCACCGGCA TCTTGAC}

DNA and RNA sequences used for PARPI activation studies. dsDNA is the 601 widom sequence: 5'-ctggagaatccc ggtgccgaggccgctcaattggtcgtagacagctctagcaccgcttaaacgcacgtacg cgctgtccccegcgttttaaccgccaaggggattactccetagtctccaggcacgtgtcagatatatacatcctgt-3' dsRNA was made from in vitro transcription of the widom sequence using the MEGAscript T7 Transcritpion kit (AM1334).

PARP-1 enzymatic assay. PARP1 (constant at $1 \mu \mathrm{M}$ ) and 'activators' (DNA or RNA; $1-2 \mu \mathrm{M}$ ) were mixed to a final volume of $20 \mu \mathrm{l}$ in $50 \mathrm{mM}$ Tris ( $\mathrm{pH} 8), 50 \mathrm{mM} \mathrm{NaCl}, 10 \mathrm{mM}$ $\mathrm{MgCl}_{2}$, and $1 \mathrm{mM}$ DTT and allowed to incubate for $1 \mathrm{~h}$ at $30^{\circ}$ C. Twenty microliters of the $\mathrm{NAD}^{+}$stock $(1 \mathrm{mM})$ were added to the above tubes for the final $500 \mu \mathrm{M}\left[\mathrm{NAD}^{+}\right]$. Reactions were quenched after $1 \mathrm{~min}$ with $5 \times$ Laemmli buffer, were immediately boiled for $3 \mathrm{~min}$, and were analyzed by $8 \%$ SDS-PAGE. Gels were stained with coomassie. If the protein is active, with $\mathrm{NAD}^{+}$in the presence of 'activators', it makes higher molecular weight smeared band.

Bioinformatic analyses. CLIP-seq analyses: Replicate libraries were multiplexed and sequenced on an Illumina Hi-seq 2500 using $100 \mathrm{bp}$ paired-end sequencing. Each library yielded between 0.9 and 97 million unique reads (Supplementary Table S1). Biological replicates were performed to avoid possible confounds in the data sets introduced by Illumina sequencing artifacts; all bioinformatics analyses were performed independently for each CLIP sample and all conclusions were independently validated for all CLIP samples. Since very similar conclusions were obtained, the replicates were combined for the subsequent analyses. Adapters and primers were trimmed from the sequences using a custom script trimAdapters.pl, which incorporated Trimmomatic v0.33 [74]. Quality control was checked using FastQC [75] v0.11.4. Trimmed reads were then concatenated as single-end reads and aligned to the human hg38 genome assembly. Reads were then aligned to the genome both without a reference transcriptome using bowtie v1.1.1 [76] and with a reference transcriptome using tophat v2.0.13 [77, 78] and the Ensembl v82 gtf [79, 80]. PAR-CLIP peaks were analyzed using PARalyzer (v1.5) [29]. The PARalyzer analysis required several steps. In addition to considering the samples independently, the samples were combined as they theoretically represent technical replicates. The first step to prepare sequences for PARalyzer was to filter the sequences using fastx_collapser v0.0.14, which is part of the FASTX Toolkit [81]. The collapsed sequences were then aligned to the human hg38 reference genome assembly using bowtie with the PARalyzer-suggested parameters. Results from PARalyzer were then parsed into evidence and sequence files using a custom script, which prepares the files for input into the motif detection program cERMIT v1.0.1 [31]. Correlation between replica was performed as follows: normalized read counts for each PAR-CLIP peak were obtained by first dividing the total number of aligned reads in the sample by one million and then dividing the number of reads for each peak by this value. A Pearson correlation was calculated on normalized read counts for intersecting peaks across the samples. Intersecting peaks were those peaks covering the same genomic location by one or more nucleotides.

Genomic feature determination. In order to assign mappings to each of the PAR-CLIP peaks (including intronic, exonic, intergenic, promoter, 5' UTR, and 3' UTR), each of these regions was marked on the hg38 assembly of the human genome using Ensembl genes and transcripts [80]. Annotations for each region were obtained using the Biomart [82] tools from Ensembl for the GrCh38 (hg38) assembly. A custom perl script was used to parse out each of these features into chromosome-specific files. Once all files were parsed, all of the regions of each chromosome were assigned to a value. The peaks (in bed format) along with the alignments for the collapsed sequences (in bowtie format) were used to create genome tracks for the UCSC genome browser (see Supplementary Data 1).

Motif analysis. K-mer enrichment motif analysis was carried out calculating 4-mer enrichments by sliding within a 20 -nt-long window along PAR-CLIP clusters and using the shuffled (10 000 times) hg38 human protein-coding open reading frames as background sequences.

Exon-intron and intron-exon boundary analyses. Exonic regions on the Homo sapiens reference genome assembly hg38 were retrieved from the Ensembl database [83]. The exonic regions were filtered to include only exons on protein-coding transcripts. In addition, duplicated exon start and end locations across transcripts for the same gene were removed from the analysis. Specifically, for the analysis of exon to intron boundaries, duplicated exon end locations on the forward DNA strand and duplicated exon start locations on the reverse DNA strand were removed. For the analysis of intron to exon boundaries, duplicated exon start locations on the forward DNA strand and duplicated exon end locations on the reverse DNA strand were removed. This resulted in 281967 unique exon-intron locations and 278090 unique intron-exon locations. A custom $\mathrm{C}++$ program was created to count the number of PAR-CLIP sequences covering each base for a distance of 100 bases in the exon and 300 bases in the intron from all exon boundaries. Two complementary methods were used to establish the number of PAR-CLIP sequences expected to cover a genomic region at random. One method utilized the Shuffle tool in the Bedtools package [84] to randomly position exon starting and ending locations while preserving the number and size of exonic regions 
on individual chromosomes. The second method utilized the Random tool in the Bedtools package to obtain 150000 random sequences of 5000 bases in length, the maximum length of the majority of intronic regions [85]. With this method, the starting location for each region represented an exon to intron boundary, and the ending location represented an intron to exon boundary. Half of the sequences were used in the analysis of exon to intron boundaries with the other half used for the analysis of intron to exon boundaries. The $\mathrm{C}++$ program mentioned above was used to count PAR-CLIP sequences covering each base for 100 bases into a simulated exonic region and 300 bases into a simulated intronic region.

RNA-seq analysis after PARP1 knockdown. RNA-seq libraries were constructed using the TruSeq stranded mRNA LT Sample preparation Kit with poly-A enrichment according to the manufacturer's instruction. The libraries corresponded to the three control samples (cells treated with non-targeting, siRNA, Dharmacon Inc.) and three PARP1 knockdown (Dharmacon Inc). These libraries were multiplexed and sequenced on the Illumina NextSeq 500 using the NextSeq 500/550 2×75 cycle High Output Kit v2 (Cat\# FC-404-2002).

Differential gene expression and alternative splicing analysis. RNA-seq reads were mapped to the hg38 reference genome assembly using tophat2 (version 2.0.13) [78], generating alignment files in bam format. PARP1 regulated differentially expressed genes were detected using the tuxedo suite of programs including cufflinks-cuffdiff2 (version 2.2.1). Differentially expressed genes were considered significant with $P$-value $\leq 0.05$ and $\mid \mathrm{FCl} \geq 1$. We next identified PARP1-regulated differential alternative splicing events corresponding to five major types of alternative splicing event patterns by rMATS (v3.2.5) [86]. For each alternative splicing event, both the reads mapped to the exon-exon junction and the reads mapped to the exon body were used as rMATS input. Putative PARP1-regulated AS events were identified as those with significant difference in inclusion levels $(|\Delta \mathrm{PSI}| \geq 5 \%)$ between knockdown and control at an false discovery rate $(\mathrm{FDR})<5 \%$.

rMAPS. In order to determine the binding patterns of PARP within significantly detected skipped exon events, the PARCLIP peaks and all detected skipped/retained exon events were used as input into the rMAPS [33] server. The rMAPS server differentiates between significant skipping and inclusion events, and determines differential binding associated with each type of event in comparison to background introns and exons.

Data deposition. RNA-seq data are deposited in GEO (GSE91051) along with the PAR-CLIP data (GSE95360). The processed files for the PARCLIP peaks, differential gene expression, and alternative splicing analysis are provided as Supplementary Data 1. Visualization tracks for PARCLIP and RNASeq data are provided as a track hub on the UCSC Genome Browser (http://bit.ly/217f5OY).

\section{Conflict of Interest}

The authors declare no conflict of interest.

\section{Acknowledgements}

This work used the Vincent J Coates Genomics Sequencing Laboratory at UC Berkeley, supported by NIH S10 Instrumentation Grants S10RR029668 and S10RR027303 for PARCLIP sequencing and University of Louisville for poly-A RNA sequencing. We also thank Dr Louis Hersh for critical review of the manuscript, Dr Fried for help with EMSA analyses. We would like to thank the Markey Cancer Center's Research Communications Office for manuscript editing and assistance with graphic design. His-tagged PARP-1 expression vectors were a kind gift from the Pascal laboratory (University of Montreal). This research was supported by NIH grants P20 GM103436 (ECR): 1RO1ES024478 and NSF MCB-1517986 (YNF-M).

\section{Author contributions}

Conceived and designed the experiments: YNF-M. Performed the experiments: MM and YNF-M. Analyzed the data: MM, JLC, ECR, and YNF-M. Contributed reagents/ materials: MM and YNF-M. Wrote the paper: MM, JLC, ECR, and YNF-M.

\section{References}

1 De Vos M, Schreiber V, Dantzer F. The diverse roles and clinical relevance of PARPs in DNA damage repair: current state of the art. Biochem Pharmacol 2012; 84: 137-146.

2 Gibson BA, Zhang Y, Jiang $\mathrm{H}$ et al. Chemical genetic discovery of PARP targets reveals a role for PARP-1 in transcription elongation. Science 2016; 353: 45-50.

3 Ji Y, Tulin AV. Post-transcriptional regulation by poly (ADP-ribosyl)ation of the RNA-binding proteins. Int J Mol Sci 2013; 14: 16168-16183.

4 Krishnakumar R, Kraus WL. PARP-1 regulates chromatin structure and transcription through a KDM5B-dependent pathway. Mol Cell 2010; 39: 736-749.

5 Petesch SJ, Lis JT. Overcoming the nucleosome barrier during transcript elongation. Trends Genet 2012; 28: 285-294.

6 Petesch SJ, Lis JT. Activator-induced spread of poly(ADPribose) polymerase promotes nucleosome loss at Hsp70. Mol Cell 2012; 45: 64-74.

7 Pinnola A, Naumova N, Shah M, Tulin AV. Nucleosomal core histones mediate dynamic regulation of poly(ADPribose) polymerase 1 protein binding to chromatin and induction of its enzymatic activity. J Biol Chem 2007; 282: 32511-32519.

8 Krishnakumar R, Gamble MJ, Frizzell KM, Berrocal JG, Kininis M, Kraus WL. Reciprocal binding of PARP-1 and histone $\mathrm{H} 1$ at promoters specifies transcriptional outcomes. Science 2008; 319: 819-821.

9 Zhang T, Berrocal JG, Yao J et al. Regulation of poly (ADP-ribose) polymerase-1-dependent gene expression through promoter-directed recruitment of a nuclear NAD+ synthase. J Biol Chem 2012; 287: 12405-12416. 
10 Gibson BA, Kraus WL. New insights into the molecular and cellular functions of poly(ADP-ribose) and PARPs. Nat Rev Mol Cell Biol 2012; 13: 411-424.

11 Hassa PO, Covic M, Hasan S, Imhof R, Hottiger MO. The enzymatic and DNA binding activity of PARP-1 are not required for NF-kappa B coactivator function. $J$ Biol Chem 2001; 276: 45588-45597.

12 Hinz M, Stilmann M, Arslan SC, Khanna KK, Dittmar G, Scheidereit C. A cytoplasmic ATM-TRAF6-cIAP1 module links nuclear DNA damage signaling to ubiquitin-mediated NF-kappaB activation. Mol Cell 2010; 40: 63-74.

13 Nasta F, Laudisi F, Sambucci M, Rosado MM, Pioli C. Increased Foxp3+ regulatory $\mathrm{T}$ cells in poly(ADP-Ribose) polymerase-1 deficiency. J Immunol 2010; 184: 3470-3477.

14 Hassa PO, Hottiger MO. The diverse biological roles of mammalian PARPS, a small but powerful family of polyADP-ribose polymerases. Front Biosci 2008; 13: 3046-3082.

15 Vyas S, Chang P. New PARP targets for cancer therapy. Nat Rev Cancer 2014; 14: 502-509.

16 Di Giammartino DC, Shi Y, Manley JL. PARP1 represses PAP and inhibits polyadenylation during heat shock. Mol Cell 2013; 49: 7-17.

17 Guetg C, Scheifele F, Rosenthal F, Hottiger MO, Santoro R. Inheritance of silent rDNA chromatin is mediated by PARP1 via noncoding RNA. Mol Cell 2012; 45: 790-800.

18 Ji Y, Tulin AV. Poly(ADP-ribosyl)ation of heterogeneous nuclear ribonucleoproteins modulates splicing. Nucleic Acids Res 2009; 37: 3501-3513.

19 Castello A, Fischer B, Eichelbaum K et al. Insights into RNA biology from an atlas of mammalian mRNA-binding proteins. Cell 2012; 149: 1393-1406.

20 Matveeva E, Maiorano J, Zhang Q et al. Involvement of PARP1 in the regulation of alternative splicing. Cell Discov 2016; 2: 15046.

21 D GH, Kelley DR, Tenen D, Bernstein B, Rinn JL. Widespread RNA binding by chromatin-associated proteins. Genome Biol 2016; 17: 28.

22 Mekishvili M, Matveeva E, Fondufe-Mittendorf Y. Methodology to Identify Poly-ADP-Ribose Polymerase 1 (PARP1)-mRNA Targets by PAR-CLiP. Methods Mol Biol 2017; 1608: 211-228,

23 Ascano M, Hafner M, Cekan P, Gerstberger S, Tuschl T. Identification of RNA-protein interaction networks using PAR-CLIP. Wiley Interdiscip Rev2012; 3: 159-177.

24 Benhalevy D, McFarland HL, Sarshad AA, Hafner M. PAR-CLIP and streamlined small RNA cDNA library preparation protocol for the identification of RNA binding protein target sites. Methods 2017; 118-119: 41-49.

25 Hafner M, Landthaler M, Burger L et al. PAR-CliP--a method to identify transcriptome-wide the binding sites of RNA binding proteins. J Vis Exp 2010; 41: 2034.

26 Spitzer J, Hafner M, Landthaler $\mathrm{M}$ et al. PAR-CLIP (photoactivatable ribonucleoside-enhanced crosslinking and immunoprecipitation): a step-by-step protocol to the transcriptome-wide identification of binding sites of RNA-binding proteins. Methods Enzymol 2014; 539: 113-161.
27 Hafner M, Landthaler M, Burger L et al. Transcriptomewide identification of RNA-binding protein and microRNA target sites by PAR-CLIP. Cell 2010; 141: 129-141.

28 Polymenidou M, Lagier-Tourenne C, Hutt KR et al. Long pre-mRNA depletion and RNA missplicing contribute to neuronal vulnerability from loss of TDP-43. Nat Neurosci 2011; 14: 459-468.

29 Corcoran DL, Georgiev S, Mukherjee N et al. PARalyzer: definition of RNA binding sites from PAR-CLIP short-read sequence data. Genome Biol 2011; 12: R79.

30 Singh G, Ricci EP, Moore MJ. RIPiT-Seq: a highthroughput approach for footprinting RNA:protein complexes. Methods 2014; 65: 320-332.

31 Georgiev S, Boyle AP, Jayasurya K, Ding X, Mukherjee S, Ohler U. Evidence-ranked motif identification. Genome Biol 2010; 11: R19.

32 Kishore S, Jaskiewicz L, Burger L, Hausser J, Khorshid M, Zavolan M. A quantitative analysis of CLIP methods for identifying binding sites of RNA-binding proteins. Nat Methods 2011; 8: 559-564.

33 Park JW, Jung S, Rouchka EC, Tseng YT, Xing Y. rMAPS: RNA map analysis and plotting server for alternative exon regulation. Nucleic Acids Res 2016; 44: W333-W338.

34 Huambachano O, Herrera F, Rancourt A, Satoh MS. Double-stranded DNA binding domain of poly(ADPribose) polymerase-1 and molecular insight into the regulation of its activity. J Biol Chem 2011; 286: 7149-7160.

35 Langelier MF, Servent KM, Rogers EE, Pascal JM. A third zinc-binding domain of human poly(ADP-ribose) polymerase-1 coordinates DNA-dependent enzyme activation. J Biol Chem 2008; 283: 4105-4114.

36 Xiao R, Tang P, Yang B et al. Nuclear matrix factor hnRNP U/SAF-A exerts a global control of alternative splicing by regulating U2 snRNP maturation. Mol Cell 2012; 45: 656-668.

37 Katz Y, Wang ET, Airoldi EM, Burge CB. Analysis and design of RNA sequencing experiments for identifying isoform regulation. Nat Methods 2010; 7: 1009-1015.

38 Lebedeva S, Jens M, Theil K et al. Transcriptome-wide analysis of regulatory interactions of the RNA-binding protein HuR. Mol Cell 2011; 43: 340-352.

39 Anko ML, Muller-McNicoll M, Brandl H et al. The RNAbinding landscapes of two SR proteins reveal unique functions and binding to diverse RNA classes. Genome Biol 2012; 13: R17.

40 Felsenfeld G. Quantitative approaches to problems of eukaryotic gene expression. Biophys Chem 2003; 100: 607-613.

41 Narlikar GJ, Fan HY, Kingston RE. Cooperation between complexes that regulate chromatin structure and transcription. Cell 2002; 108: 475-487.

42 Mitchell PJ, Tjian R. Transcriptional regulation in mammalian cells by sequence-specific DNA binding proteins. Science 1989; 245: 371-378.

43 Tuck AC, Tollervey D. A transcriptome-wide atlas of RNP composition reveals diverse classes of mRNAs and lncRNAs. Cell 2013; 154: 996-1009. 
44 Baltz AG, Munschauer M, Schwanhausser B et al. The mRNA-bound proteome and its global occupancy profile on protein-coding transcripts. Mol Cell 2012; 46: 674-690.

45 Khalil AM, Guttman M, Huarte M et al. Many human large intergenic noncoding RNAs associate with chromatin-modifying complexes and affect gene expression. Proc Natl Acad Sci USA 2009; 106: 11667-11672.

46 Agirre E, Bellora N, Allo M et al. A chromatin code for alternative splicing involving a putative association between CTCF and HP1alpha proteins. BMC Biol 2015; 13: 31.

47 Braunschweig U, Gueroussov S, Plocik AM, Graveley BR, Blencowe BJ. Dynamic integration of splicing within gene regulatory pathways. Cell 2013; 152: 1252-1269.

48 Bentley DL. Coupling mRNA processing with transcription in time and space. Nat Rev Genet 2014; 15: 163-175.

49 Le Hir H, Seraphin B. EJCs at the heart of translational control. Cell 2008; 133: 213-216.

50 Volanakis A, Passoni M, Hector RD et al. Spliceosomemediated decay (SMD) regulates expression of nonintronic genes in budding yeast. Genes Dev 2013; 27: 2025-2038.

51 Egecioglu DE, Kawashima TR, Chanfreau GF. Quality control of MATa1 splicing and exon skipping by nuclear RNA degradation. Nucleic Acids Res 2012; 40: 1787-1796.

52 Sayani S, Chanfreau GF. Sequential RNA degradation pathways provide a fail-safe mechanism to limit the accumulation of unspliced transcripts in Saccharomyces cerevisiae. RNA 2012; 18: 1563-1572.

53 Garneau NL, Wilusz J, Wilusz CJ. The highways and byways of mRNA decay. Nat Rev Mol Cell Biol 2007; 8: 113-126.

54 Schmid M, Jensen TH. The exosome: a multipurpose RNA-decay machine. Trends Biochem Sci 2008; 33: 501-510.

55 Houseley J, Tollervey D. The many pathways of RNA degradation. Cell 2009; 136: 763-776.

56 Sayani S, Janis M, Lee CY, Toesca I, Chanfreau GF. Widespread impact of nonsense-mediated mRNA decay on the yeast intronome. Mol Cell 2008; 31: 360-370.

57 Kawashima T, Douglass S, Gabunilas J, Pellegrini M, Chanfreau GF. Widespread use of non-productive alternative splice sites in Saccharomyces cerevisiae. PLoS Genet 2014; 10: e1004249.

58 Frischmeyer PA, Dietz HC. Nonsense-mediated mRNA decay in health and disease. Hum Mol Genet 1999; 8: 1893-1900.

59 Jungmichel S, Rosenthal F, Altmeyer M, Lukas J, Hottiger MO, Nielsen ML. Proteome-wide identification of poly(ADP-Ribosyl)ation targets in different genotoxic stress responses. Mol Cell 2013; 52: 272-285.

60 Gagne JP, Isabelle M, Lo KS et al. Proteome-wide identification of poly(ADP-ribose) binding proteins and poly (ADP-ribose)-associated protein complexes. Nucleic Acids Res 2008; 36: 6959-6976.

61 Isabelle M, Moreel X, Gagne JP et al. Investigation of PARP-1, PARP-2, and PARG interactomes by affinitypurification mass spectrometry. Proteome Sci 2010; 8: 22.

62 Malanga M, Czubaty A, Girstun A, Staron K, Althaus FR. Poly(ADP-ribose) binds to the splicing factor ASF/SF2 and regulates its phosphorylation by DNA topoisomerase I. $J$ Biol Chem 2008; 283: 19991-19998.

63 Vidakovic M, Gluch A, Qiao J et al. PARP-1 expression in the mouse is controlled by an autoregulatory loop: PARP-1 binding to an upstream S/MAR element and to a novel recognition motif in its promoter suppresses transcription. $J$ Mol Biol 2009; 388: 730-750.

64 Lodhi N, Kossenkov AV, Tulin AV. Bookmarking promoters in mitotic chromatin: poly(ADP-ribose)polymerase-1 as an epigenetic mark. Nucleic Acids Res 2014; 42: 7028-7038.

65 Cogoi S, Xodo LE. Enhanced G4-DNA binding of 5,10,15,20 (N-propyl-4-pyridyl) porphyrin (TPrPyP4): a comparative study with TMPyP4. Chem Commun (Camb) 2010; 46: 7364-7366.

66 Soldatenkov VA, Vetcher AA, Duka T, Ladame S. First evidence of a functional interaction between DNA quadruplexes and poly(ADP-ribose) polymerase-1. ACS Chem Biol 2008; 3: 214-219.

67 Fekete A, Kenesi E, Hunyadi-Gulyas E et al. The guaninequadruplex structure in the human c-myc gene's promoter is converted into B-DNA form by the human poly(ADPribose)polymerase-1. PLoS ONE 2012; 7: e42690.

68 Bock FJ, Todorova TT, Chang P. RNA regulation by poly(ADP-ribose) polymerases. Mol Cell 2015; 58: 959-969.

69 Atasheva S, Frolova EI, Frolov I. Interferon-stimulated poly(ADP-Ribose) polymerases are potent inhibitors of cellular translation and virus replication. J Virol 2014; 88: 2116-2130.

70 Petesch SJ, Lis JT. Rapid, transcription-independent loss of nucleosomes over a large chromatin domain at Hsp70 loci. Cell 2008; 134: 74-84.

71 Langelier MF, Planck JL, Servent KM, Pascal JM. Purification of human PARP-1 and PARP-1 domains from Escherichia coli for structural and biochemical analysis. Methods Mol Biol 2011; 780: 209-226.

72 Fried M, Crothers DM. Equilibria and kinetics of lac repressor-operator interactions by polyacrylamide gel electrophoresis. Nucleic Acids res 1981; 9: 6505-6525.

73 Fried MG, Daugherty MA. Electrophoretic analysis of multiple protein-DNA interactions. Electrophoresis 1998; 19: $1247-1253$.

74 Bolger AM, Lohse M, Usadel B. Trimmomatic: a flexible trimmer for Illumina sequence data. Bioinformatics 2014; 30: $2114-2120$.

75 Andrews S. FatsQC: A Quality Control Tool for High Throughput Sequence Data. Available from http://www. bioinformatics.babraham.ac.uk/projects/fastqc/.

76 Langmead B, Trapnell C, Pop M, Salzberg SL. Ultrafast and memory-efficient alignment of short DNA sequences to the human genome. Genome Biol 2009; 10: R25.

77 Trapnell C, Roberts A, Goff L et al. Differential gene and transcript expression analysis of RNA-seq experiments with TopHat and Cufflinks. Nat Protoc 2012; 7: 562-578.

78 Kim D, Pertea G, Trapnell C, Pimentel H, Kelley R, Salzberg SL. TopHat2: accurate alignment of transcriptomes in the presence of insertions, deletions and gene fusions. Genome Biol 2013; 14: R36. 
79 Cunningham F, Amode MR, Barrell D et al. Ensembl 2015. Nucleic Acids Res 2015; 43: D662-D669.

80 Flicek P, Amode MR, Barrell D et al. Ensembl 2014. Nucleic Acids Res 2014; 42: D749-D755.

81 Gordon, A., and Hannon, GJ FASTX Toolkit. Available online at http://hannonlab.cshl.edu/fastx_toolkit 2010.

82 Kasprzyk A. BioMart: driving a paradigm change in biological data management. Database the journal of biological databases and curation 2011; 2011: bar049.

83 Hubbard T, Barker D, Birney E et al. The Ensembl genome database project. Nucleic Acids Res 2002; 30: 38-41.

84 Quinlan AR, Hall IM. BEDTools: a flexible suite of utilities for comparing genomic features. Bioinformatics 2010; 26: 841-842.

85 Lander ES, Linton LM, Birren B et al. Initial sequencing and analysis of the human genome. Nature 2001; 409: 860-921.

86 Shen S, Park JW, Lu ZX et al. rMATS: robust and flexible detection of differential alternative splicing from replicate
RNA-Seq data. Proc Natl Acad Sci USA 2014; 111: E5593-E5601.

(Supplementary information is linked to the online version of the paper on the Cell Discovery website.)

(c) (i) This work is licensed under a Creative Commons Attribution 4.0 International License. The images or other third party material in this article are included in the article's Creative Commons license, unless indicated otherwise in the credit line; if the material is not included under the Creative Commons license, users will need to obtain permission from the license holder to reproduce the material. To view a copy of this license, visit http://creativecommons.org/licenses/by/4.0/

(C) The Author(s) 2017 